\title{
Time evolution of the radio continuum of young starbursts: the importance of synchrotron emission
}

\author{
H. Hirashita ${ }^{1}$ and L. K. Hunt ${ }^{2}$ \\ ${ }^{1}$ Center for Computational Sciences, University of Tsukuba, Tsukuba, Ibaraki 305-8577, Japan \\ e-mail: hirasita@ccs.tsukuba.ac.jp \\ 2 INAF - Istituto di Radioastronomia-Sezione Firenze, Largo E. Fermi 5, 50125 Firenze, Italy \\ e-mail: hunt@arcetri.astro.it
}

Received 18 May 2006 / Accepted 30 August 2006

\section{ABSTRACT}

\begin{abstract}
We investigate the radio spectral energy distributions (SEDs) of young star-forming galaxies and how they evolve with time. The duration and luminosity of the nonthermal radio emission from supernova remnants (SNRs) are constrained by using the observational radio SEDs of SBS 0335-052 and IZw 18, which are the two lowest-metallicity blue compact dwarf galaxies in the nearby universe. The typical radio "fluence" for SNRs in SBS 0335-052, that is the radio energy emitted per SNR over its radiative lifetime, is estimated to be $\sim 6-22 \times 10^{22} \mathrm{~W} \mathrm{~Hz}^{-1} \mathrm{yr}$ at $5 \mathrm{GHz}$. On the other hand, the radio fluence in I Zw 18 is $\sim 1-3 \times 10^{22} \mathrm{~W} \mathrm{~Hz}^{-1} \mathrm{yr}$ at $5 \mathrm{GHz}$. We discuss the origin of this variation and propose scaling relations between synchrotron luminosity and gas density. We have also predicted the time dependence of the radio spectral index and of the spectrum itself, for both the "active" (SBS 0335-052) and "passive" (IZw 18) cases. These models enable us to roughly age date and classify radio spectra of star-forming galaxies into active/passive classes. Implications for high- $z$ galaxy evolution are also discussed.
\end{abstract}

Key words. galaxies: dwarf - galaxies: evolution - galaxies: ISM - ISM: supernova remnants - radio continuum: galaxies

\section{Introduction}

Radio emission from galaxies is known to be connected with star formation activity. Two radiative processes are responsible: thermal free-free radiation from ionized gas in H II regions and synchrotron radiation from relativistic electrons spiraling in magnetic fields (Condon 1992). The former originates from ionized gas around massive stars, while the latter comes from supernova remnants (SNRs) whose progenitors are massive stars. Since massive stars have short lifetimes, both thermal and nonthermal emission should trace the current star formation rate (SFR).

Nevertheless, the physical basis of the radio emission is not fully established. The observed radio flux in luminous evolved starbursts and normal spiral galaxies is dominated by nonthermal emission, but not from the short timescale radiation of discrete SNRs; more than $90 \%$ of it is probably due to the diffusion of cosmic ray electrons in the galaxy disk (Condon 1992) over timescales of $10^{7}-10^{8} \mathrm{yr}$ (Helou \& Bicay 1993). Indeed, if the theoretically estimated adiabatic timescale (e.g., Woltjer 1972) and the observationally obtained $\Sigma-D$ (surface brightness vs. diameter) relation for discrete SNRs (e.g., Clark \& Caswell 1976) are used to derive the nonthermal emission in galaxies (e.g., Biermann 1976; Ulvestad 1982), the emission is underpredicted by a factor of 10 or so.

In very young galaxies with ages $\lesssim 10 \mathrm{Myr}$, however, the cosmic-ray diffusion mechanisms have not yet had a chance to dominate the radio emission. Nonthermal synchrotron radiation from discrete SNRs would be expected to dominate over the diffuse component. Moreover, in galaxies with starbursts younger than $\sim 3 \mathrm{Myr}$, there should be very little synchrotron emission since the SNRs have not yet exploded. Indeed, some galaxies with a deficit of nonthermal emission are observed
(Roussel et al. 2003) and appear to be due to starbursts observed within a few Myr of their onset, after a long period ( $~(100 \mathrm{Myr})$ of quiescence.

In this paper, we investigate theoretically the time evolution of the radio spectral energy distribution (SED) in young starforming galaxies. In principle, this is a straightforward process because we know that the radio SED can be determined by the star formation history, for which we have already developed the formalism (Hirashita et al. 2002; Hirashita \& Hunt 2004). In practice, it is a difficult exercise because of the unknown nature of the time evolution of the nonthermal radio component. Hence, we adopt nearby blue compact dwarf galaxies (BCDs) as an observational sample to constrain our theoretical model of radio emission. Most BCDs have a young age of current star formation (although most of them had previous starburst episodes), and a low metallicity (e.g., Izotov \& Thuan 2004). This implies that they are relatively unevolved chemically and may provide a reasonable template to investigate star formation properties of young galaxies. The BCDs as a class have radio spectra with different properties than those of evolved luminous star-forming galaxies (Klein et al. 1991); flatter radio spectra of BCDs indicate that they have a lower fraction of nonthermal emission than more luminous systems. Hence, they may lack the diffuse emission that characterizes the radio spectrum of larger disk galaxies, and thus are ideal targets for constraining our models.

Two classes of star-formation activity in BCDs have recently emerged observationally, as proposed by Hunt et al. (2003a) and Hunt \& Hirashita (2006, in preparation). They argue that the star-formation modes in the two most metal-poor galaxies, SBS 0335-052 and I Zw 18, are very different, in spite of their similar metallicities $(12+\log (\mathrm{O} / \mathrm{H})=7.3$ and $12+\log (\mathrm{O} / \mathrm{H})=7.2$, respectively; Skillman \& Kennicutt 1993; Izotov et al. 1999). 
The major star-forming region of SBS 0335-052 is compact and dense (radius $r_{\mathrm{SF}} \lesssim 40 \mathrm{pc}$, number density $n \gtrsim 600 \mathrm{~cm}^{-3}$; Dale et al. 2001; Izotov \& Thuan 1999). Moreover, SBS 0335-052 hosts several super star clusters (SSCs), detectable $\mathrm{H}_{2}$ emission lines in the near-infrared (NIR) (Vanzi et al. 2000), a large dust extinction $\left(A_{V} \sim 16 \mathrm{mag}\right.$; Thuan et al. 1999; Hunt et al. 2001; Plante \& Sauvage 2002), and high dust temperature (Hunt et al. 2001; Dale et al. 2001; Takeuchi et al. 2003). On the contrary, the star-forming regions in IZw 18 are diffuse $\left(r_{\mathrm{SF}} \gtrsim 100 \mathrm{pc}\right.$, $n \lesssim 100 \mathrm{~cm}^{-3}$ ), and contain no SSCs. NIR $\mathrm{H}_{2}$ emission has not been detected (Hunt et al., private communication), and the dust extinction is moderate $\left(A_{V} \sim 0.2 \mathrm{mag}\right.$; Cannon et al. 2002). We consider a region with such properties as "passive" following Hunt et al. (2003a), while a SBS 0335-052-like starforming property is considered "active". The similar metallicities of SBS 0335-052 (active) and IZw 18 (passive) imply that the chemical abundance is not a primary factor in determining the star-forming properties. We argue that the compactness of star-forming regions, which affect gas density, gas dynamics, and so on, is important in the dichotomy of active and passive modes.

In this paper, we extend this active-passive classification into the radio regime by focusing on SBS 0335-052 and IZw 18 as representatives of metal-poor BCDs, and also of two starforming modes in BCDs: "active" and "passive". This paper is organized as follows. First, in Sect. 2 we explain the model that describes the evolution of radio SEDs. Some basic results are given in Sect. 3, where observational data of SBS 0335-052 and IZw 18 are compared with the results for active and passive modes. The constraints obtained here are compared with theoretical descriptions of the physical processes of synchrotron radiation in Sect. 4. In Sect. 5 we generalize the results of our models with predictions for the time evolution of radio SEDs distinguishing between active and passive modes, and discuss implications for high-redshift star formation. Finally we give our conclusions in Sect. 6.

\section{Model description}

The radio emission from galaxies is interpreted to be composed of two components (Condon 1992): thermal free-free (bremsstrahlung) emission and nonthermal synchrotron radiation. The former requires treatment of ionized regions around star-forming regions and the latter is related to SNRs. Our models include these processes as described below. We approximate the dominant star-forming region in a BCD to a single zone with spherical symmetry.

\subsection{Thermal component}

The free-free radiation in ionized regions is responsible for the flat thermal component of radio SEDs. The luminosity of the thermal component is proportional to the number of ionizing photons (with energy larger than $13.6 \mathrm{eV}$ ) emitted per unit time, $\dot{N}_{\text {ion }}$. In order to calculate $\dot{N}_{\text {ion }}$, we need the stellar ionizing photon luminosity and the stellar lifetime.

We use the fitting formulae of Schaerer (2002) for the stellar lifetime $\tau_{m}$ (denoted as $t_{\star}$ in Schaerer 2002) and the number of ionizing photons emitted per unit time $Q(m)$ (see Table 6 of Schaerer 2002). Those quantities are calculated as functions of stellar mass at the zero age main sequence, $m$. We adopt $Z=0$ (zero metallicity) for the stellar properties because we treat a metal-poor phase. If we adopt the solar metallicity instead, $\dot{N}_{\text {ion }}$ is $\sim 2$ times smaller (Schaerer 2002). Considering those two extreme metallicities, we consider that the uncertainty caused by the stellar metallicity is within a factor of 2 .

The evolution of $\dot{N}_{\text {ion }}$ as a function of time $t$ is calculated by

$\dot{N}_{\text {ion }}(t)=\int_{0}^{\infty} \mathrm{d} m \int_{0}^{\tau_{m}} \mathrm{~d} t^{\prime} Q(m) \phi(m) \psi\left(t-t^{\prime}\right)$,

where $\psi(t)$ is the SFR at $t$, and $\phi(m)$ is the initial mass function (IMF; the definition of the IMF is the same as that in Tinsley 1980). In this paper, we assume a Salpeter IMF $\phi(m) \propto m^{-2.35}$ (Salpeter 1955) with stellar mass range of $0.1-100 M_{\odot}$. With this IMF, the mass fraction of stars more massive than $5 M_{\odot}$ is 0.18 . The calculated radio luminosities are roughly scaled with this fraction of massive stars if another IMF is adopted. The functional form of the SFR $\psi(t)$ is specified in Sect. 2.3.

The number of ionizing photons can be related to the thermal radio luminosity at the frequency $v, L_{\mathrm{th}}^{0}(v)$ as (Hunt et al. 2004, hereafter H04; valid for $10000 \mathrm{~K} \lesssim T \lesssim 20000 \mathrm{~K}$ )

$$
\begin{aligned}
L_{\mathrm{th}}^{0}(v)= & 1.58 \times 10^{19} \frac{n\left(\mathrm{H}^{+}\right)+n\left(\mathrm{He}^{+}\right)}{n\left(\mathrm{H}^{+}\right)}\left(\frac{v}{1 \mathrm{GHz}}\right)^{-0.1} \\
& \times\left(\frac{T}{10^{4} \mathrm{~K}}\right)^{-0.31}\left(\frac{\dot{N}_{\text {ion }}}{10^{52} \mathrm{~s}^{-1}}\right) \mathrm{W} \mathrm{Hz}^{-1}
\end{aligned}
$$

where $n\left(\mathrm{H}^{+}\right)$and $n\left(\mathrm{He}^{+}\right)$are the number densities of ionized hydrogen and ionized helium, respectively (we assume $n\left(\mathrm{He}^{+}\right)=$ $0.08 n\left(\mathrm{H}^{+}\right)$in this paper; $\left.\mathrm{H} 04\right)$, and $T$ is the gas temperature. For the gas temperature in the ionized region, we assume a typical value of metal-poor $\mathrm{H}$ II regions: $T \simeq 2 \times 10^{4} \mathrm{~K}$ (Skillman $\&$ Kennicutt 1993; Izotov et al. 1999). The change of the temperature from $2 \times 10^{4} \mathrm{~K}$ to $1 \times 10^{4} \mathrm{~K}$ has only a minor effect on $L_{\text {th }}^{0}$ and causes a factor of 2.5 difference in the free-free optical depth estimated below (Eq. (3)).

A part of the radio emission is self-absorbed by the ionized gas. We estimate the optical depth of the free-free radiation, $\tau_{\mathrm{ff}}$ as

$$
\tau_{\mathrm{ff}} \simeq 0.328\left(\frac{T}{10^{4} \mathrm{~K}}\right)^{-1.35}\left(\frac{E M}{10^{6} \mathrm{pc} \mathrm{cm}^{-6}}\right)\left(\frac{v}{\mathrm{GHz}}\right)^{-2.1}
$$

(H04), where the emission measure $E M$ is estimated by assuming a constant electron number density $n_{\mathrm{e}}: E M=n_{\mathrm{e}}^{2} \ell(\ell$ is the length of the ionized region along the line of sight). The length $\ell$ is estimated by dividing the volume with the surface of the ionized region. We thus obtain the emission measure as

$E M=\frac{4}{3} n_{\mathrm{e}}^{2} r_{\mathrm{i}}$

where $r_{\mathrm{i}}$ is the radius of ionized region defined in Sect. 2.4. In the ionized region, the following condition is assumed to be satisfied for the charge neutrality (H04):

$n_{\mathrm{e}}=n\left(\mathrm{H}^{+}\right)+n\left(\mathrm{He}^{+}\right) \simeq 1.08 n_{\mathrm{H}}$,

where $n_{\mathrm{H}}$ is the number of hydrogen nuclei. Since the ionized gas is responsible for both emission and absorption, it is reasonable to assume that the absorbing medium is intermixed with the emitting material. In this case, the monochromatic luminosity of the thermal component emerging from the ionized region becomes

$L_{\mathrm{th}}(v)=\frac{1-\exp \left(-\tau_{\mathrm{ff}}\right)}{\tau_{\mathrm{ff}}} L_{\mathrm{th}}^{0}(v)$ 


\subsection{Nonthermal component}

Nonthermal radio emission from galaxies originates from SNRs. Since we consider young star-forming regions, we only treat Type II supernovae (SNe II), whose progenitors have short lifetimes $^{1}$. The rate of SNe II as a function of time, $\gamma(t)$, is given by

$\gamma(t)=\int_{8 M_{\odot}}^{\infty} \psi\left(t-\tau_{m}\right) \phi(m) \mathrm{d} m$.

First we use a "standard" estimate for the intrinsic nonthermal radio luminosity at frequency $v, L_{\mathrm{nt}, \mathrm{s}}^{0}(v)$ by relating it to $\gamma(t)$ (e.g., Condon 1992):

$$
\begin{aligned}
L_{\mathrm{nt}, \mathrm{s}}^{0}(v)= & 1.2 \times 10^{22}\left(\frac{E_{\mathrm{SN}}}{10^{51} \mathrm{erg}}\right)^{-1 / 17}\left(\frac{n_{\mathrm{H}}}{1 \mathrm{~cm}^{-3}}\right)^{-2 / 17} \\
& \times\left(\frac{\gamma(t)}{1 \mathrm{yr}^{-1}}\right)\left(\frac{v}{408 \mathrm{MHz}}\right)^{\alpha} \mathrm{W} \mathrm{Hz}^{-1}
\end{aligned}
$$

where $E_{\mathrm{SN}}$ is the explosion energy of a SN II $\left(E_{\mathrm{SN}}=10^{51} \mathrm{erg}\right.$ is assumed in this paper), and $\alpha$ is the spectral index. We designate this model for the nonthermal component as the "s-model" ("s" stands for standard). However, this formula is obtained by assuming the $\Sigma-D$ (surface brightness vs. diameter) relation derived from the Galactic SNRs (Clark \& Caswell 1976; Ulvestad 1982) and the adiabatic lifetime $\tau_{\text {ad }}$ as time during which the remnant emits in the radio. The adiabatic timescale is estimated as (Woltjer 1972; Condon 1992):

$\tau_{\mathrm{ad}} \simeq 3.4 \times 10^{4}\left(\frac{E_{\mathrm{SN}}}{10^{51} \mathrm{erg}}\right)^{4 / 17}\left(\frac{n_{\mathrm{H}}}{1 \mathrm{~cm}^{-3}}\right)^{-9 / 17} \mathrm{yr}$.

The SN II rate inferred from the nonthermal radio luminosities by using Eq. (8) is generally too high (Condon 1992 and references therein; see also Sect. 1). In other words, the radio luminosity given by the dependence on $\gamma$ in Eq. (8) is an underestimate. Thus, we need to reexamine the underlying physical assumptions. Condon (1992) suggests that the discrepancy arises because SNRs produce more than $90 \%$ of the nonthermal radio emission long after the individual SNRs have faded out and the electrons have diffused throughout the galaxy. However, this may not be true in young starbursts, hence warranting an investigation of the possibility that individual SNRs have a radio lifetime longer than the adiabatic timescale (Ilovaisky \& Lequeux 1972).

In order to constrain the radio energy emitted by a SNR over its entire lifetime - the "fluence" or time-luminosity integral we treat the duration $\left(\tau_{\mathrm{nt}}\right)$ and the luminosity at a frequency $v_{0}\left(l_{\mathrm{nt}}\right)$ as parameters to be determined from observational constraints. Then, the nonthermal luminosity is written as

$L_{\mathrm{nt}, \mathrm{p}}^{0}(v)=l_{\mathrm{nt}} \tau_{\mathrm{nt}} \gamma\left(v / v_{0}\right)^{\alpha}$.

This model of the nonthermal component is called "p-model" ("p" stands for parameterization). We take $v_{0}=5 \mathrm{GHz}$ unless otherwise stated. We adopt a typical spectral index of the Galactic SNRs: $\alpha=-0.5$ (Clark \& Caswell 1976). Hunt et al. (2005) mention that $\alpha=-0.4$ and -0.5 fit the radio spectrum of IZw 18 but that the fit is significantly degraded for $\alpha=-0.8$.

After accounting for the absorption, the nonthermal radio component emitted by the star-forming region is estimated as

$L_{\mathrm{nt}}(v)=E\left(\tau_{\mathrm{ff}}\right) L_{\mathrm{nt}, i}^{0}(v)$,

\footnotetext{
${ }^{1}$ Although see Mannucci et al. (2005) for the idea that Type Ia supernovae are important in young systems.
}

where $E\left(\tau_{\mathrm{ff}}\right)$ is the escaping fraction of the radio photons, and $i$ is the label indicating " $s$ " for the s-model and " $p$ " for the p-model. We consider two cases for $E\left(\tau_{\mathrm{ff}}\right)$ depending on the geometry of the absorbing material relative to the radiation source. One is the "screen geometry", where the absorbing material surrounds the radiation source, and the other is the "mixed geometry", where the source and the absorber are mixed homogeneously:

$E\left(\tau_{\mathrm{ff}}\right)= \begin{cases}E_{\mathrm{sc}}\left(\tau_{\mathrm{ff}}\right) \equiv \exp \left(-\tau_{\mathrm{ff}}\right) & (\text { screen }), \\ E_{\mathrm{mix}}\left(\tau_{\mathrm{ff}}\right) \equiv \frac{1-\exp \left(-\tau_{\mathrm{ff}}\right)}{\tau_{\mathrm{ff}}} & (\text { mixed }) .\end{cases}$

\subsection{Star formation rate}

Stars form as a result of the gravitational collapse of a gas cloud. Therefore, it is physically reasonable to relate the SFR with the free-fall (or dynamical) timescale of gas (Elmegreen 2000). We consider a star-forming region with an initial number density of neutral hydrogen $n_{\mathrm{H} 0}$. The free-fall time, $t_{\mathrm{ff}}$, is estimated as

$t_{\mathrm{ff}}=\sqrt{\frac{3 \pi}{32 G \rho}} \simeq \frac{4.35 \times 10^{7}}{\sqrt{n_{\mathrm{H} 0}}} \mathrm{yr}$,

where $G$ is the gravitational constant, and $\rho$ is the mass density of the gas. In Eq. (13), we have used $\rho=\mu m_{\mathrm{H}} n_{\mathrm{H} 0}$, where $m_{\mathrm{H}}$ is the mass of a hydrogen atom, and the factor $\mu$ is for the correction for helium. We assume that $\mu=1.4$ in this paper.

The SFR, $\psi(t)$, basically scales with the gas mass divided by the free-fall time:

$\psi(t)=\frac{\epsilon_{\mathrm{SF}} M_{\mathrm{gas}}}{t_{\mathrm{ff}}} f(t)$,

where $\epsilon_{\mathrm{SF}}$ is the star formation efficiency defined as the conversion efficiency of a gas into stars over a free-fall time, $M_{\text {gas }}$ is the total gas mass available in the star-forming region, and the nondimensional function $f(t)$ specifies the functional form of the SFR. Numerically, the SFR is estimated as

$$
\begin{aligned}
\psi(t) \simeq & 0.230\left(\frac{\epsilon_{\mathrm{SF}}}{0.1}\right)\left(\frac{M_{\mathrm{gas}}}{10^{7} M_{\odot}}\right)\left(\frac{n_{\mathrm{H} 0}}{100 \mathrm{~cm}^{-3}}\right)^{1 / 2} \\
& \times f(t) M_{\odot} \mathrm{yr}^{-1} .
\end{aligned}
$$

We set the zero point of time $t$ at the onset of star formation in the star-forming region. We define the termination time of star formation as $t_{\mathrm{i}}$ such that $r_{\mathrm{i}}\left(t_{\mathrm{i}}\right)=r_{\mathrm{SF}}$, where $r_{\mathrm{SF}}$ is the radius of the star-forming region (i.e., $t_{\mathrm{i}}$ is the time when the entire starforming region is ionized; see Sect. 2.4 for the definition of $r_{\mathrm{i}}$ ). If the entire region is never ionized, $t_{\mathrm{i}}$ is taken to be infinity. We note that $t_{\mathrm{ff}}<t_{\mathrm{i}}$ is satisfied for the conditions adopted in this paper. Then, we investigate the following functional form for $f(t)$ :

$f(t)=f_{\mathrm{c}}(t) \equiv \begin{cases}\exp \left(-\epsilon_{\mathrm{SF}} t / t_{\mathrm{ff}}\right) & \text { if } 0 \leq t \leq t_{\mathrm{i}}, \\ 0 & \text { if } t<0 \text { or } t>t_{\mathrm{i}},\end{cases}$

which is called "continuous SFR". In order to represent an extreme starburst, we also adopt another functional form for the SFR:

$f(t)=f_{\mathrm{b}}(t) \equiv\left\{\begin{array}{l}s \text { if } 0 \leq t \leq t_{\mathrm{ff}}, \\ 0 \text { if } t<0 \text { or } t>t_{\mathrm{ff}},\end{array}\right.$

where the constant $s$ is adjusted to satisfy

$$
\begin{aligned}
s & =\frac{1}{t_{\mathrm{ff}}} \int_{0}^{t_{\mathrm{i}}} \exp \left(-\epsilon_{\mathrm{SF}} t / t_{\mathrm{ff}}\right) \mathrm{d} t \\
& =\frac{1}{\epsilon_{\mathrm{SF}}}\left[1-\exp \left(-\epsilon_{\mathrm{SF}} t_{\mathrm{i}} / t_{\mathrm{ff}}\right)\right],
\end{aligned}
$$


where $t_{\mathrm{i}}$ is determined by the continuous SFR model. This assures that the total stellar mass formed in a star-forming region is the same between the two models. In the burst model, all the star formation occurs on a dynamical timescale. For the star formation efficiency, we assign a value $\epsilon_{\mathrm{SF}}=0.1$ (e.g., Inoue et al. 2000).

The initial hydrogen number density can be related to the gas mass in the star-forming region as

$\frac{4 \pi}{3} r_{\mathrm{SF}}^{3} \mu m_{\mathrm{H}} n_{\mathrm{H} 0}=M_{\mathrm{gas}}$.

Then, the numerical value of the number density is estimated as

$n_{\mathrm{H} 0} \simeq 69\left(\frac{r_{\mathrm{SF}}}{100 \mathrm{pc}}\right)^{-3}\left(\frac{M_{\mathrm{gas}}}{10^{7} M_{\odot}}\right) \mathrm{cm}^{-3}$.

\subsection{Radius of ionized region}

In order to treat the range of evolution variations from deeply embedded H II regions to normal H II regions, it is crucial to include pressure-driven expansion of $\mathrm{H}$ II regions. To focus on the radio SED, we adopt a simple analytical approximation, which divides the evolution of an $\mathrm{H}$ II region into two stages: the first stage is the growth of ionizing front due to the increase of ionizing photons, and the second is the pressure-driven expansion of ionized gas. The expansion speed of ionizing front in the first stage is simply estimated by the increasing rate of the Strömgren radius. The Strömgren radius $r_{\mathrm{S} 0}$ under the initial density is defined by the following relation:

$\frac{4 \pi}{3} r_{\mathrm{S} 0}^{3} n_{\mathrm{e}} n_{\mathrm{H} 0} \alpha^{(2)}=\dot{N}_{\text {ion }}$,

where $\alpha^{(2)}$ is the recombination coefficient excluding captures to the ground ( $n=1)$ level, and $n_{\mathrm{e}}$ is estimated by Eq. (5) with $n_{\mathrm{H}}=n_{\mathrm{H} 0}$. The increase of $r_{\mathrm{S} 0}$ is caused by the accumulation of ionizing stars. Roughly speaking, as long as $\dot{r}_{\mathrm{S} 0}$ (the increase rate of the Strömgren radius) is larger than the sound speed of ionized gas, $C_{\mathrm{II}}$ (we assume $C_{\mathrm{II}}=10 \mathrm{~km} \mathrm{~s}^{-1}$ in this paper), the ionizing front propagates before the system responds hydrodynamically. Therefore, we neglect the hydrodynamical expansion if $\dot{r}_{\mathrm{S} 0}>$ $C_{\mathrm{II}}$, and assume that the radius of ionized region is equal to the Strömgren radius: $r_{\mathrm{i}}=r_{\mathrm{S} 0}$.

Once $\dot{r}_{\mathrm{S}}<C_{\mathrm{II}}$ is satisfied, pressure-driven expansion is treated. Since the density evolves, we define the Strömgren radius $r_{\mathrm{S}}$ under the current density:

$\frac{4 \pi}{3} r_{\mathrm{S}}^{3} n_{\mathrm{e}} n_{\mathrm{H}} \alpha^{(2)}=\dot{N}_{\text {ion }}$

In this situation, the growth of the ionizing region is governed by the pressure of ionized gas and the luminosity change of the central stars has minor effects. Therefore, the following equation derived by assuming a constant luminosity (Spitzer 1978) approximately holds:

$\dot{r}_{\mathrm{i}}=C_{\mathrm{II}} \sqrt{\rho_{\mathrm{II}} / \rho_{\mathrm{I}}}$,

where $\rho_{\mathrm{I}}$ and $\rho_{\mathrm{II}}$ are the gas densities outside and inside of the ionized region, respectively. Here, $\rho_{\mathrm{II}} / \rho_{\mathrm{I}}$ is approximately estimated by

$\frac{\rho_{\mathrm{II}}}{\rho_{\mathrm{I}}} \simeq\left(\frac{r_{\mathrm{i} 0}}{r_{\mathrm{i}}}\right)^{3 / 2}$, where $r_{\mathrm{i} 0}$ is evaluated by the Strömgren radius under the current ionizing photon luminosity and the gas density of $\rho_{\mathrm{I}}$. Since $n_{\mathrm{H}}$ is defined in the ionized region, we relate it with $\rho_{\text {II }}$ as

$\rho_{\mathrm{II}}=\mu m_{\mathrm{H}} n_{\mathrm{H}}$.

The density in the neutral region is assumed to be constant:

$\rho_{\mathrm{I}}=\mu m_{\mathrm{H}} n_{\mathrm{H} 0}$.

Equations (23) and (24) are numerically integrated to obtain $r_{\mathrm{i}}$ as a function of $t$. For the convenience of interpretation, some approximate analytical solutions are described in Appendix A.

The dynamical expansion is treated as long as $r_{\mathrm{i}}<r_{\mathrm{S}}$. When the SFR declines significantly, $r_{\mathrm{S}}$ begins to decrease. Thus, $r_{\mathrm{i}}>$ $r_{\mathrm{S}}$ can be realized at a certain time. When $r_{\mathrm{i}}>r_{\mathrm{S}}, r_{\mathrm{i}}$ is replaced with $r_{\mathrm{S}}$ and $n_{\mathrm{H}}$ is fixed; that is, we finish treating the dynamical expansion.

\section{Observational constraints}

Here we use observations to constrain some of the physical parameters in our models. In particular, the radiative energy -timeluminosity integral- of the nonthermal synchrotron component is the most important parameter to be obtained in this paper. Two representative metal-poor BCDs, SBS 0335-052 and IZw 18 (Hirashita \& Hunt 2004), are used here. In our previous works (Hirashita et al. 2002; Hirashita \& Hunt 2004), SBS 0335-052 has been used as a proxy for a genuinely young galaxy in which the mass of underlying old population is negligible (Vanzi et al. 2000). The same may be true for I Zw 18 in which the mass fraction due to an underlying evolved stellar population ( $\gtrsim 2 \mathrm{Gyr})$ is not more than $\sim 20 \%$ (Hunt et al. 2003b). We adopt $54.3 \mathrm{Mpc}$ for the distance of SBS 0335-052 (Thuan et al. 1997) and $12.6 \mathrm{Mpc}$ for IZw 18 (Östlin 2000).

We calculate the time dependence of the radio SED, $L(v)$, by summing the thermal and nonthermal components:

$L(v)=L_{\mathrm{th}}(v)+L_{\mathrm{nt}}(v)$.

Each of the following three items has two selections as summarized in Table 1:

(i) nonthermal emission of a SNR: standard / parameterized (Sect. 2.2);

(ii) optical depth of nonthermal emission: screen / mixed (Sect. 2.2);

(iii) SFR: continuous/burst (Sect. 2.3).

In total we have 8 models, which are labeled as models $\mathrm{A}-\mathrm{H}$ as shown in Table 1.

\subsection{SBS 0335-052}

We adopt $r_{\mathrm{SF}}=20 \mathrm{pc}$ and $n_{\mathrm{H} 0}=7 \times 10^{3} \mathrm{~cm}^{-3}$ (i.e., $M_{\mathrm{gas}}=$ $\left.8.1 \times 10^{6} M_{\odot} ; t_{\mathrm{ff}}=5.2 \times 10^{5} \mathrm{yr}\right)$. We selected these values after surveying the full range of parameters so that the observable quantities (SFR, $n_{\mathrm{H}}$, and $E M$ ) are consistent with observations at the age estimated by Vanzi et al. (2000). The time evolution of the relevant quantities is shown below to verify whether those values indeed reproduce the observations. The radius of the starforming region is also consistent with Takeuchi et al. (2005), who reproduce the observational FIR SED of SBS 0335-052.

First, we evaluate the continuous SFR. The time evolution of $r_{\mathrm{i}}, n_{\mathrm{H}}, E M$, and SFR is shown in Figs. 1a,b,c, and d, respectively. The SFR averaged for $5 \mathrm{Myr}$ is $\simeq 0.8 M_{\odot} \mathrm{yr}^{-1}$ with the total stellar 

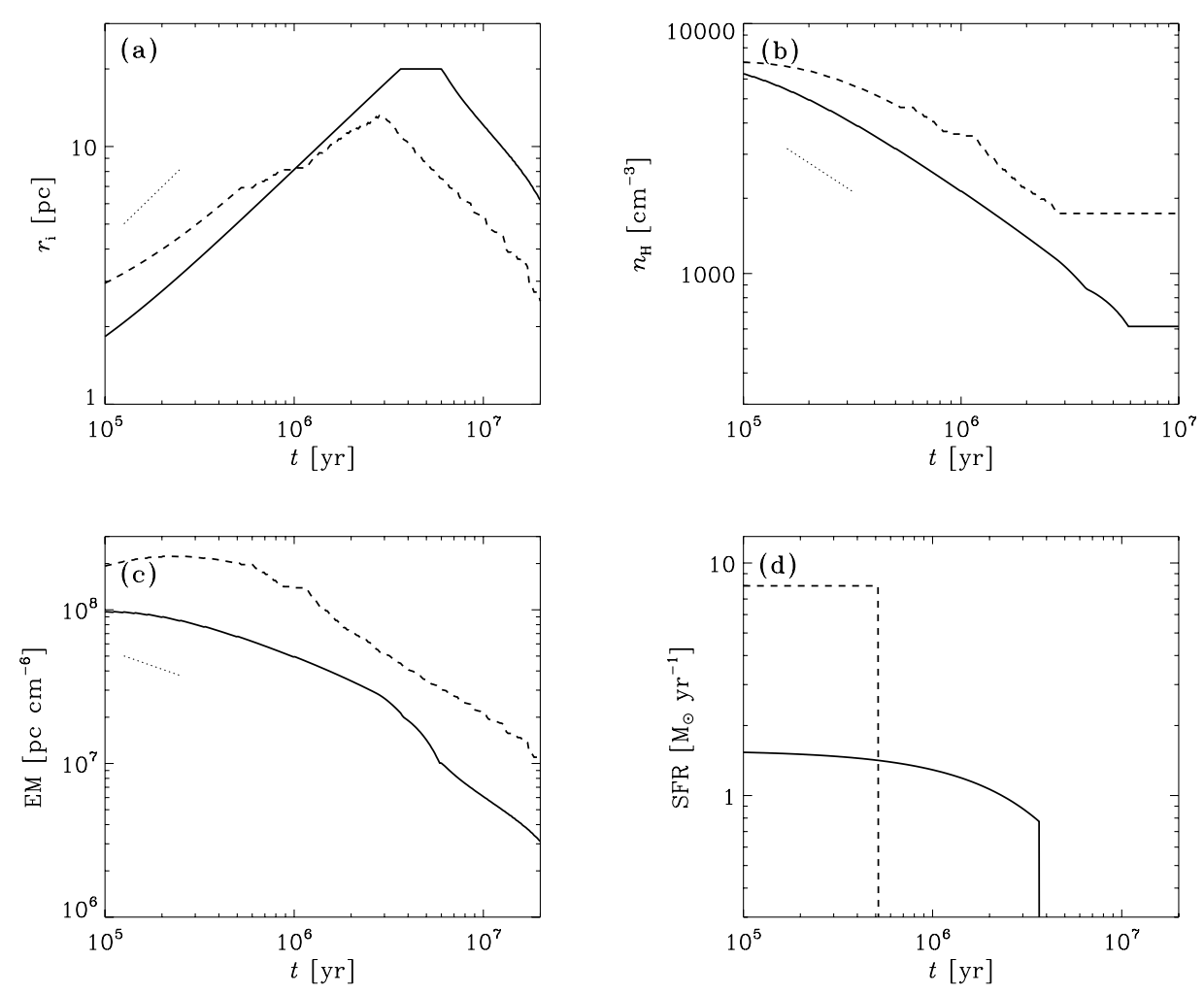

Fig. 1. Time evolution of basic quantities concerning radio emission, with the solid and dashed lines corresponding to the continuous and burst SFR, respectively. The initial conditions are selected to be the "active" mode, consistent with the observations of SBS 0335-052 at $t \simeq 5$ Myr. a) Radius of ionized region $r_{\mathrm{i}}$ as a function of time. b) Number density of protons in the ionized region $n_{\mathrm{H}}$. c) Emission measure. d) Star formation rate. The dotted lines show the analytical approximation: $r_{\mathrm{i}} \propto t^{5 / 7}, n_{\mathrm{H}} \propto t^{-4 / 7}$, and $E M \propto t^{-3 / 7}$.

Table 1. Models of radio SEDs.

\begin{tabular}{cccc}
\hline \hline Model & $L_{\mathrm{nt}}^{0}{ }^{a}$ & $E\left(\tau_{\mathrm{ff}}\right)^{b}$ & $\mathrm{SFR}^{c}$ \\
\hline $\mathrm{A}$ & $\mathrm{s}$ & $\mathrm{s}$ & $\mathrm{c}$ \\
$\mathrm{B}$ & $\mathrm{s}$ & $\mathrm{s}$ & $\mathrm{b}$ \\
$\mathrm{C}$ & $\mathrm{s}$ & $\mathrm{m}$ & $\mathrm{c}$ \\
$\mathrm{D}$ & $\mathrm{s}$ & $\mathrm{m}$ & $\mathrm{b}$ \\
$\mathrm{E}$ & $\mathrm{p}$ & $\mathrm{s}$ & $\mathrm{c}$ \\
$\mathrm{F}$ & $\mathrm{p}$ & $\mathrm{s}$ & $\mathrm{b}$ \\
$\mathrm{G}$ & $\mathrm{p}$ & $\mathrm{m}$ & $\mathrm{c}$ \\
$\mathrm{H}$ & $\mathrm{p}$ & $\mathrm{m}$ & $\mathrm{b}$ \\
\hline
\end{tabular}

a See Sect. 2.2. The standard and parameterized models are denoted as "s" and "p", respectively (Eqs. (8) and (10), respectively).

$b$ See Sect. 2.2. The screen and mixed models are denoted as "s" and "m", respectively (Eq. (12)).

$c$ See Sect. 2.3. The continuous and burst SFRs are denoted as "c" and "b", respectively (Eqs. (16) and (17), respectively).

mass formed of $4 \times 10^{6} M_{\odot}$. The SFR of stars more massive than $5 M_{\odot}\left(S F R_{>5} M_{\odot}\right)$, which is less affected by the assumed IMF, is estimated to be $0.14 M_{\odot} \mathrm{yr}^{-1}$, which is consistent with the estimate by $\mathrm{H} 04\left(S F R_{>5} M_{\odot} \simeq 0.13-0.15 M_{\odot} \mathrm{yr}^{-1}\right)$. At $t=$ $5 \mathrm{Myr}, n_{\mathrm{H}} \simeq 7 \times 10^{2} \mathrm{~cm}^{-3}$, which is also compatible with the observational results (Izotov et al. 1999; H04).

The radius of the ionized region $r_{\mathrm{i}}$ monotonically increases until $t=3.7$ Myr because of the pressure-driven expansion and the increase of $\dot{N}_{\text {ion }}$. At $t=3.7 \mathrm{Myr}$, the entire star-forming region is ionized. Until then, the increasing behavior of $r_{\mathrm{i}}$ is approximated by $r_{\mathrm{i}} \propto t^{5 / 7}$ as derived in Appendix A. In Fig. 1a, we show the slope of $r_{\mathrm{i}} \propto t^{5 / 7}$ (dotted line), which is roughly consistent with the calculated result (solid line). The initial evolution of density is also explained by the analytical relation derived in Appendix as shown in Fig. $1 \mathrm{~b}\left(n_{\mathrm{H}} \propto t^{-4 / 7}\right)$. As a result, the emission measure decreases as $E M \propto t^{-3 / 7}$ as shown in Fig. 1c.

If we adopt the burst SFR ( $s=5.1$; Eq. (18)), we obtain the time evolutions as depicted in Fig. 1. As mentioned above, value of $s$ is determined to ensure that the total stellar mass formed is the same between the burst and continuous modes. Since the star formation stops on a short timescale, the driving force of the dynamical expansion decreases more rapidly than in the case of the continuous SFR. Thus, the maximum radius of the ionized region is smaller and the density is kept higher. This high density makes the $E M$ higher in the burst SFR, and the $E M$ derived observationally by $\mathrm{H} 04\left(E M=3-7 \times 10^{7} \mathrm{pc} \mathrm{cm}^{-6}\right)$ is more consistent with the burst SFR than with the continuous SFR at $t \sim 5$ Myr. Although the burst scenario is more consistent with the observations of SBS 0335-052, in what follows we examine the two cases as extremes for the SFR.

We show the radio SEDs calculated by various models listed in Table 1, and examine whether or not the model predictions agree with observational data. The radio data are shown in Fig. 2: the squares are from H04, and the cross is from Dale et al. (2001). The SED at $t=5 \mathrm{Myr}$ is presented in Fig. 2, where the dotted and dashed lines represent the nonthermal and thermal components, respectively. The sum of those two components is shown by the solid line in each panel. The panel (a) shows the result of model A, in which we adopt the "standard" model for the nonthermal radio emission. In this case, the thermal emission dominates the radio SED. The model 

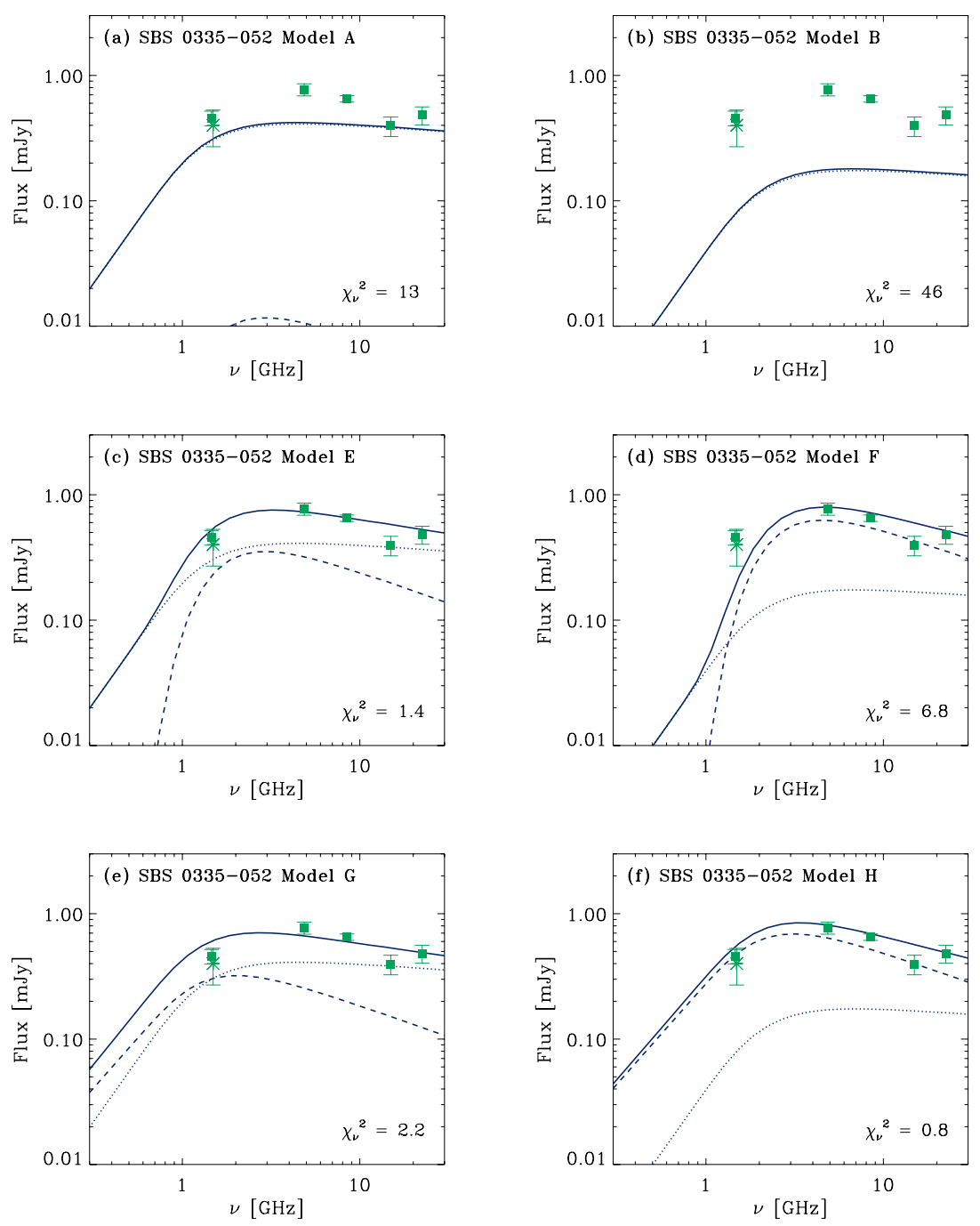

Fig. 2. Radio SEDs at $t=5 \mathrm{Myr}$ calculated by a) model A, b) model B, c) model E, d) model F, e) model G, and f) model $\mathrm{H}$ for the same initial condition as Fig. 1. For model E, we adopt the best fit parameter of $l_{\mathrm{nt}} \tau_{\mathrm{nt}}$. The dotted and dashed lines represent the thermal and nonthermal components, respectively, and the solid line shows the sum of those two components. The points are observational data taken from Hunt et al. (2004) (squares) and Dale et al. (2001) (cross). The reduced $\chi^{2}$ value $\left(\chi_{v}^{2}\right)$ is also shown in each panel.

prediction is inconsistent with the observational SED in the following two aspects: (i) the theoretical flux is systematically smaller than the observed one; (ii) the flat SED, dominated by the free-free component, is inconsistent with the observational SED at $v \gtrsim 4 \mathrm{GHz}$, which requires a prominent nonthermal contribution. Indeed H04 show that the nonthermal fraction of the radio luminosity at $5 \mathrm{GHz}$ is $\sim 0.7$. If we adopt model $\mathrm{B}$ with a burst SFR instead of model A, the thermal component decreases, because the mean age of the stars is older. In this case also, however, the nonthermal radiation of model $\mathrm{B}$ falls well below the data presented in Fig. 2. As long as we adopt the "standard" nonthermal component (i.e., models A-D), we cannot explain the prominent nonthermal contribution. Therefore, we exclude models A-D, and adopt the parameterized model for the nonthermal component (i.e., models E-H).

In the parameterized model of the nonthermal component, $l_{\mathrm{nt}} \tau_{\mathrm{nt}}$ is treated as a parameter to be determined from observations (Eq. (10)). Hence, we estimate $l_{\mathrm{nt}} \tau_{\mathrm{nt}}$ by using the observational data of SBS 0335-052 taken by H04 and Dale et al. (2001). We adopt the same initial conditions and parameters as above, and assume an age of $t=5 \mathrm{Myr}$; we then search for the
Table 2. Best fit parameters for the nonthermal component (SBS 0335-052).

\begin{tabular}{cccc}
\hline \hline Model & $\begin{array}{c}l_{\mathrm{nt}} \tau_{\mathrm{nt}}(5 \mathrm{GHz}) \\
\left(\mathrm{W} \mathrm{Hz}^{-1} \mathrm{yr}\right)\end{array}$ & $\chi^{2}$ & $\chi_{v}^{2}$ \\
\hline $\mathrm{E}$ & $7.6 \times 10^{22}$ & 6.8 & 1.4 \\
$\mathrm{~F}$ & $22 \times 10^{22}$ & 34 & 6.8 \\
$\mathrm{G}$ & $5.8 \times 10^{22}$ & 11 & 2.2 \\
$\mathrm{H}$ & $19 \times 10^{22}$ & 4.0 & 0.8 \\
\hline
\end{tabular}

value of $l_{\mathrm{nt}} \tau_{\mathrm{nt}}$ in models E-H which minimizes $\chi^{2}$ for the six data points. The best-fit $l_{\mathrm{nt}} \tau_{\mathrm{nt}}$ are listed in Table 2 with $\chi^{2}$ and $\chi_{v}^{2}$ (reduced $\chi^{2}$; the number of freedom is five), and the best-fit SEDs for models E-H are shown in Figs. 2c-f, respectively.

The burst models require higher $l_{\mathrm{nt}} \tau_{\mathrm{nt}}$. In the burst models, all the stars form at the beginning, so that the mean stellar age is older. Thus, the thermal emission of the burst models is fainter than that of the continuous star formation models. This is why we require a higher nonthermal time-luminosity integral of a SN II in the burst SFR models (models F and H) than in the 
continuous SFR models ( $E$ and $G$ ) to explain the observed radio emission. With the nonthermal component comparable to or larger than the thermal component, the points at 4.86, 8.46, and $22.5 \mathrm{GHz}$ are well reproduced. The spectral slope of the nonthermal component is also consistent with the observations. Thus nonthermal radio emission comparable to or stronger than the thermal radio emission is required even in this young $(<5 \mathrm{Myr})$ galaxy. In general, the fits are satisfactory for models E-H except for the point at $14.9 \mathrm{GHz}$. The fractions of nonthermal emission are $0.44,0.79,0.37$, and 0.79 at $5 \mathrm{GHz}$ in models $\mathrm{E}, \mathrm{F}, \mathrm{G}$, and $\mathrm{H}$, respectively. The fractions in models $\mathrm{F}$ and $\mathrm{H}$ are more consistent with the value of 0.7 at $5 \mathrm{GHz}$ inferred from the spectral decomposition by $\mathrm{H} 04$.

The nonthermal feature of the radio spectrum is inconsistent with an age of $t<3.5 \mathrm{Myr}$, because of the time required for the onset of SNe. On the other hand, as long as we adopt an age between $4 \mathrm{Myr}$ and $7 \mathrm{Myr}$, the difference in the derived $l_{\mathrm{nt}} \tau_{\mathrm{nt}}$ is within a factor of $\sim 2$ (i.e., within the uncertainty among models E-H.) Thus, we conclude that $l_{\mathrm{nt}} \tau_{\mathrm{nt}}$ derived here is robust against the age uncertainty.

The nonthermal radiative energy emitted at $v=5 \mathrm{GHz}$ over the entire lifetime of a SNR is predicted to be $5.8-22 \times$ $10^{22} \mathrm{~W} \mathrm{~Hz}^{-1} \mathrm{yr}$ (Table 2). This is more than an order of magnitude larger than the value used in the "standard" model, in which the nonthermal radio energy emitted by a SNR is estimated based on the observed $\Sigma-D$ (surface luminosity vs. radius) relation and the theoretical adiabatic lifetime. That assumption leads to the total radio energy per unit frequency as (assuming $\alpha=-0.5$ )

$$
\begin{aligned}
E_{\mathrm{nt}}^{\mathrm{s}}(v)= & 1.1 \times 10^{29} E_{51}^{-1 / 17}\left(\frac{n_{\mathrm{H}}}{1 \mathrm{~cm}^{-3}}\right)^{-2 / 17} \\
& \times\left(\frac{v}{5 \mathrm{GHz}}\right)^{-0.5} \mathrm{~J} \mathrm{~Hz}^{-1}
\end{aligned}
$$

while our new result suggests it to be

$E_{\mathrm{nt}}^{\mathrm{p}}(v)=(1.8-7.1) \times 10^{30}\left(\frac{v}{5 \mathrm{GHz}}\right)^{-0.5} \mathrm{~J} \mathrm{~Hz}^{-1}$.

Integrating those quantities from 0.1 to $100 \mathrm{GHz}$, we obtain the total radio energy per SNR as

$$
\begin{aligned}
\mathcal{E}^{\mathrm{s}}(0.1-100 \mathrm{GHz})= & 4.7 \times 10^{39} E_{51}^{-1 / 17} \\
& \times\left(\frac{n_{\mathrm{H}}}{1 \mathrm{~cm}^{-3}}\right)^{-2 / 17} \mathrm{~J}
\end{aligned}
$$

for the "standard" case, and

$\mathcal{E}^{\mathrm{p}}(0.1-100 \mathrm{GHz})=(8-31) \times 10^{40} \mathrm{~J}$

for the "parameterized" case.

\section{2. $I Z w 18$}

We now apply our model to another "template" of nearby young BCDs, IZw 18, whose nebular oxygen abundance is low $(12+\log (\mathrm{O} / \mathrm{H})=7.2$, Skillman \& Kennicutt 1993). The star-forming region of IZw 18 is more diffuse than that of SBS 0335-052 (e.g., Hunt et al. 2003a). Thus, the different gas density of IZw 18 from SBS 0335-052 may provide us with independent information on the density dependence of radio emission.

For the initial density and the radius, we assume $n_{\mathrm{H} 0}=$ $100 \mathrm{~cm}^{-3}$ and $r_{\mathrm{SF}}=100 \mathrm{pc}$, consistently with optical spectra and images (Skillman \& Kennicutt 1993; Hunt et al. 2003a;
Hirashita \& Hunt 2004). The resulting gas mass is $M_{\mathrm{gas}}=$ $1.4 \times 10^{7} M_{\odot}$ with $t_{\mathrm{ff}}=4.4 \times 10^{6} \mathrm{yr}$; the gas mass is comparable to the observationally determined values by van Zee et al. (1998) $\left(2.6 \times 10^{7} M_{\odot}\right.$ for their H I-A component) and Lequeux $\&$ Viallefond (1980) $\left(2.1 \times 10^{7} M_{\odot}\right.$ for their component 1$)$.

We adopt an age of $10-15 \mathrm{Myr}$ for the dominant burst in I Zw 18. This is consistent with the results of our model of infrared dust emission (Hirashita \& Hunt 2004), which gives an age of 10-15 Myr and consistent with the observed metallicity and dust abundance. Takeuchi et al. (2003) show that the dust mass derived by Cannon et al. (2002) is consistent with an age of 10-30 Myr, which is also consistent with the observational upper limits of infrared flux. Recchi et al. (2004) (and references therein) argue that stars older than 0.5-1 Gyr, if any, do not produce a significant contribution to the metal budget of IZw 18, but intermediate age stars with an age of a few hundred Myr may be required from studies of the chemical abundances. An analysis of the color-magnitude diagram by Aloisi et al. (1999) also suggests the existence of intermediate-age populations. Hunt et al. (2003b) derive an age of $<500$ Myr for the oldest stellar population (but see Östlin 2000) and $\lesssim 15$ Myr for the youngest one in the main body.

First, we evaluate a continuous SFR. The time evolution of $r_{\mathrm{i}}, n_{\mathrm{H}}, E M$, and SFR is shown in Figs. 3a-d. In the first $1.4 \mathrm{Myr}$, the expansion speed of the ionizing front is faster than the sound speed because of small gas density. Thus, the dynamical response of the system begins later compared to SBS 0335-052. Moreover, because of the low density, the emission measure remains smaller than in SBS 0335-052. The star formation stops at $t=7.9 \mathrm{Myr}$ because the entire star-forming region is ionized. The total stellar mass formed is $2.4 \times 10^{6} M_{\odot}$.

In the burst SFR, the SFR is constant up to $t_{\text {dyn }}=4.4 \mathrm{Myr}$, a little shorter than the above duration (7.9 Myr). Thus, the expansion of the ionized region stops earlier and $n_{\mathrm{H}}$ remains higher. However, the decline of $r_{\mathrm{i}}$ occurs earlier because of the shorter duration, and the resulting $E M$ is roughly similar to that for the continuous SFR. As a result, there is little difference in the evolution of emission measure between the continuous SFR and the burst SFR in a low-density regime.

We also calculate radio SEDs with various models listed in Table 1. As mentioned above, we adopt an age of 10-20 Myr. Because the (continuous mode of) star formation stops at $t=$ 7.9 Myr, the radio emission decreases between $t=10$ and 20 Myr. At $10 \mathrm{Myr}$, the thermal component is too high to be consistent with the observations reported by Hunt et al. (2005). The age should be older than $12 \mathrm{Myr}$ to be consistent with the data. At $15 \mathrm{Myr}$, the thermal component is well below the observations. Thus, we examine the two ages, $t=12 \mathrm{Myr}$ and $15 \mathrm{Myr}$, as high and low limiting luminosities for the thermal component. The emission measures at $t=12 \mathrm{Myr}$ and $15 \mathrm{Myr}$ are $2.4 \times 10^{5} \mathrm{pc} \mathrm{cm}^{-6}$ and $1.4 \times 10^{5} \mathrm{pc} \mathrm{cm}^{-6}$, respectively. Those are larger than the $E M$ estimated by Hunt et al. (2005) $\left(E M \sim 10^{4} \mathrm{pc} \mathrm{cm}^{-6}\right)$, but smaller than their extreme case $\left(E M \sim 10^{7} \mathrm{pc} \mathrm{cm}^{-6}\right)$. If we adopt an age of $\gtrsim 20 \mathrm{Myr}$, the emission measure becomes too small to be consistent with observations.

Now we examine the radio SED at $t=12 \mathrm{Myr}$, but consider only the p-models for the nonthermal component, since the s-models are rejected after the investigation of SBS 0335-052. In fact, the s-models are also unacceptable for IZw 18. As in Sect. 3.1, the best-fit nonthermal time-integrated luminosity (fluence) per SN II at $5 \mathrm{GHz}\left(l_{\mathrm{nt}} \tau_{\mathrm{nt}}\right)$ is sought by minimizing $\chi^{2}$ for the six data points adopted from Hunt et al. (2005) and references therein. The data are shown in Figs. 4 and 5: the lower 

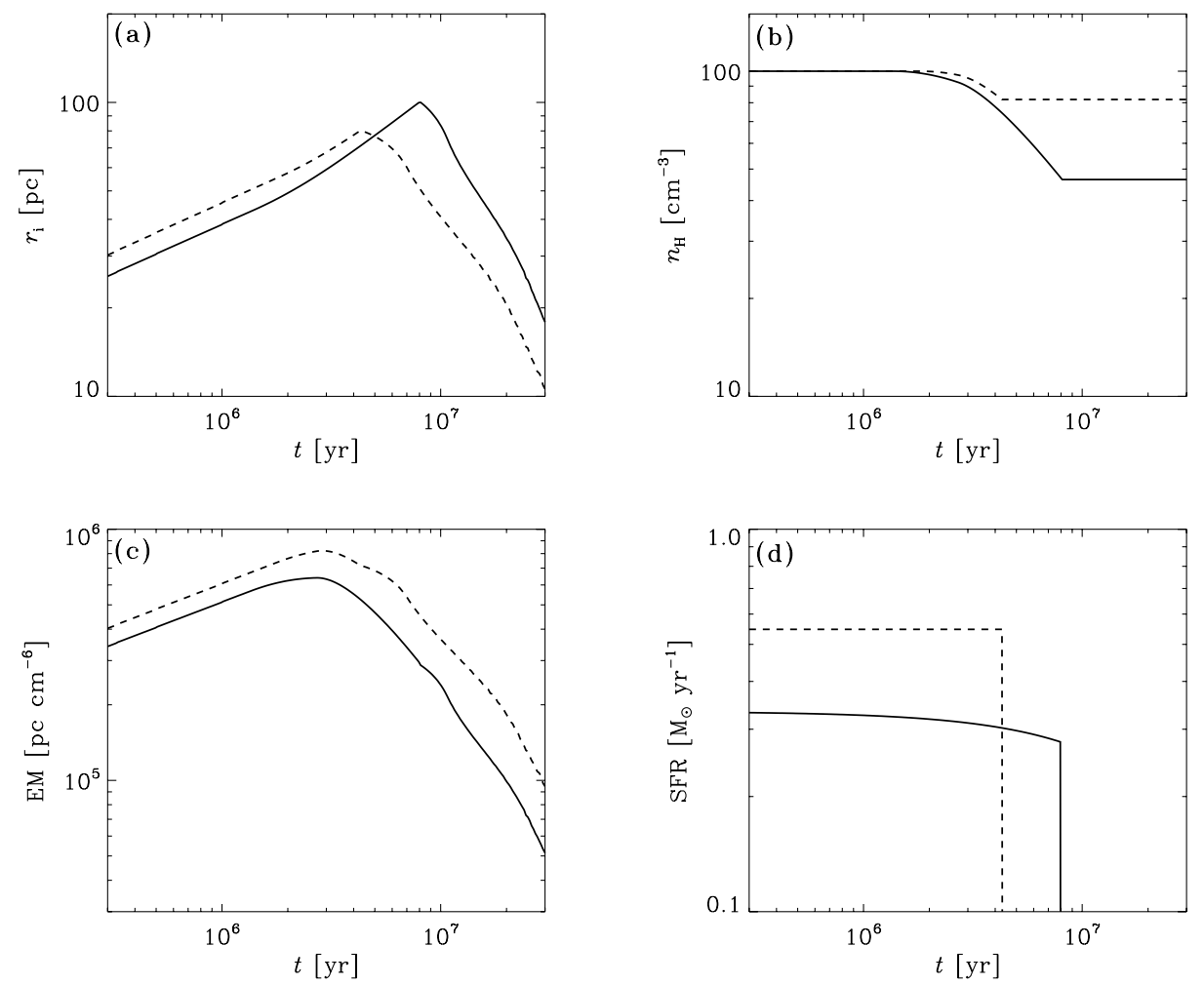

Fig. 3. Same as Fig. 1 but for the IZw 18 "passive" model.

Table 3. Best fit parameters for the nonthermal component (IZw 18 at $t=12 \mathrm{Myr}$ ).

\begin{tabular}{cccc}
\hline \hline Model & $\begin{array}{c}l_{\mathrm{nt}} \tau_{\mathrm{nt}}(5 \mathrm{GHz}) \\
\left(\mathrm{W} \mathrm{Hz}^{-1} \mathrm{yr}\right)\end{array}$ & $\chi^{2}$ & $\chi_{v}^{2}$ \\
\hline $\mathrm{E}$ & $1.1 \times 10^{22}$ & 8.2 & 1.6 \\
$\mathrm{~F}$ & $2.5 \times 10^{22}$ & 7.5 & 1.5 \\
$\mathrm{G}$ & $1.1 \times 10^{22}$ & 8.2 & 1.6 \\
$\mathrm{H}$ & $2.5 \times 10^{22}$ & 7.6 & 1.5 \\
\hline
\end{tabular}

three data points are from the VLA (Hunt et al. 2005), while the upper three are from single-dish radio telescopes (Klein et al. 1991). Although the discrepancy between those two data sets may arise from the sensitivity for the diffuse component, the discrepancy is only $\sim 40 \%$ at $v \sim 5 \mathrm{GHz}$. Thus, the following conclusions are not changed by the uncertainty in the diffuse component.

We include all the six data points to obtain the best-fit values for $l_{\mathrm{nt}} \tau_{\mathrm{nt}}$, which are shown in Table 3 for $t=12 \mathrm{Myr}$, and in Table 4 for $t=15$ Myr. Applying the values reported in those tables, we examine the radio SEDs. The results are shown in Figs. 4 and 5 for $t=12$ and $15 \mathrm{Myr}$, respectively. Those figures confirm that the nonthermal luminosity derived from the parameters in Tables 3 and 4 is consistent with the data points. Moreover, the spectral index at $v \lesssim 5 \mathrm{GHz}$ is reproduced by introducing a significant contribution from the nonthermal component.

The spectral decomposition by Hunt et al. (2005) gives a nonthermal fraction of 0.59 at $5 \mathrm{GHz}$, corresponding to a thermal flux of $0.54 \mathrm{mJy}$. This is approximately realized for models $\mathrm{F}$ and $\mathrm{H}$ shown in Panels b) and d) of Fig. 4, and for models $\mathrm{E}$ and $\mathrm{G}$ shown in Panels a) and c) of Fig. 5. However, other
Table 4. Best fit parameters for the nonthermal component (IZw 18 at $t=15 \mathrm{Myr})$.

\begin{tabular}{cccc}
\hline \hline Model & $\begin{array}{c}l_{\mathrm{nt}} \tau_{\mathrm{nt}}(5 \mathrm{GHz}) \\
\left(\mathrm{W} \mathrm{Hz}^{-1} \mathrm{yr}\right)\end{array}$ & $\chi^{2}$ & $\chi_{v}^{2}$ \\
\hline $\mathrm{E}$ & $2.4 \times 10^{22}$ & 7.7 & 1.5 \\
$\mathrm{~F}$ & $3.0 \times 10^{22}$ & 8.5 & 1.7 \\
$\mathrm{G}$ & $2.4 \times 10^{22}$ & 7.7 & 1.5 \\
$\mathrm{H}$ & $3.0 \times 10^{22}$ & 8.6 & 1.7 \\
\hline
\end{tabular}

solutions with a thermal component of 0.3-1 mJy are also permitted. The younger age of $12 \mathrm{Myr}$ gives $0.20 M_{\odot} \mathrm{yr}^{-1}$ for the averaged SFR, while $15 \mathrm{Myr}$ gives $0.16 M_{\odot} \mathrm{yr}^{-1}$. The massive $\mathrm{SFR}$ is estimated as $\mathrm{SFR}_{>5 M_{\odot}} \simeq 0.036$ and $0.029 M_{\odot} \mathrm{yr}^{-1}$, respectively. Those values are 1.5-2 times larger than the estimate of Hunt et al. (2005). If we assume a smaller SFR according to Hunt et al. (2005), $l_{\mathrm{nt}} \tau_{\mathrm{nt}}$ required to fit the observational SED becomes 1.5-2 times smaller.

Cannon et al. (2005) also provide fluxes of IZw 18 at $v=$ $1.4 \mathrm{GHz}$ and $v=8.4 \mathrm{GHz}$; their fluxes are lower than the values given by Hunt et al. (2005), but it is possible that the discrepancy comes from the sensitivity to the diffuse component. However, they also derive a spectral index that indicates a prominent nonthermal contribution. Consequently, our conclusion that a strong contribution from the nonthermal component is required should be robust (and $l_{\mathrm{nt}} \tau_{\mathrm{nt}}$ derived by us should be relatively secure).

We have derived values of $l_{\mathrm{nt}} \tau_{\mathrm{nt}}$ for IZw 18 between $1.1 \times$ $10^{22} \mathrm{~W} \mathrm{~Hz}^{-1} \mathrm{yr}$ and $2.5 \times 10^{22} \mathrm{~W} \mathrm{~Hz}^{-1} \mathrm{yr}$ at $5 \mathrm{GHz}$. Since the radio spectrum is dominated by the nonthermal component already at $15 \mathrm{Myr}$, those derived values should be robust for older ages, because the thermal component steeply declines thereafter. On the other hand, if we adopt an age smaller than $10 \mathrm{Myr}$, 

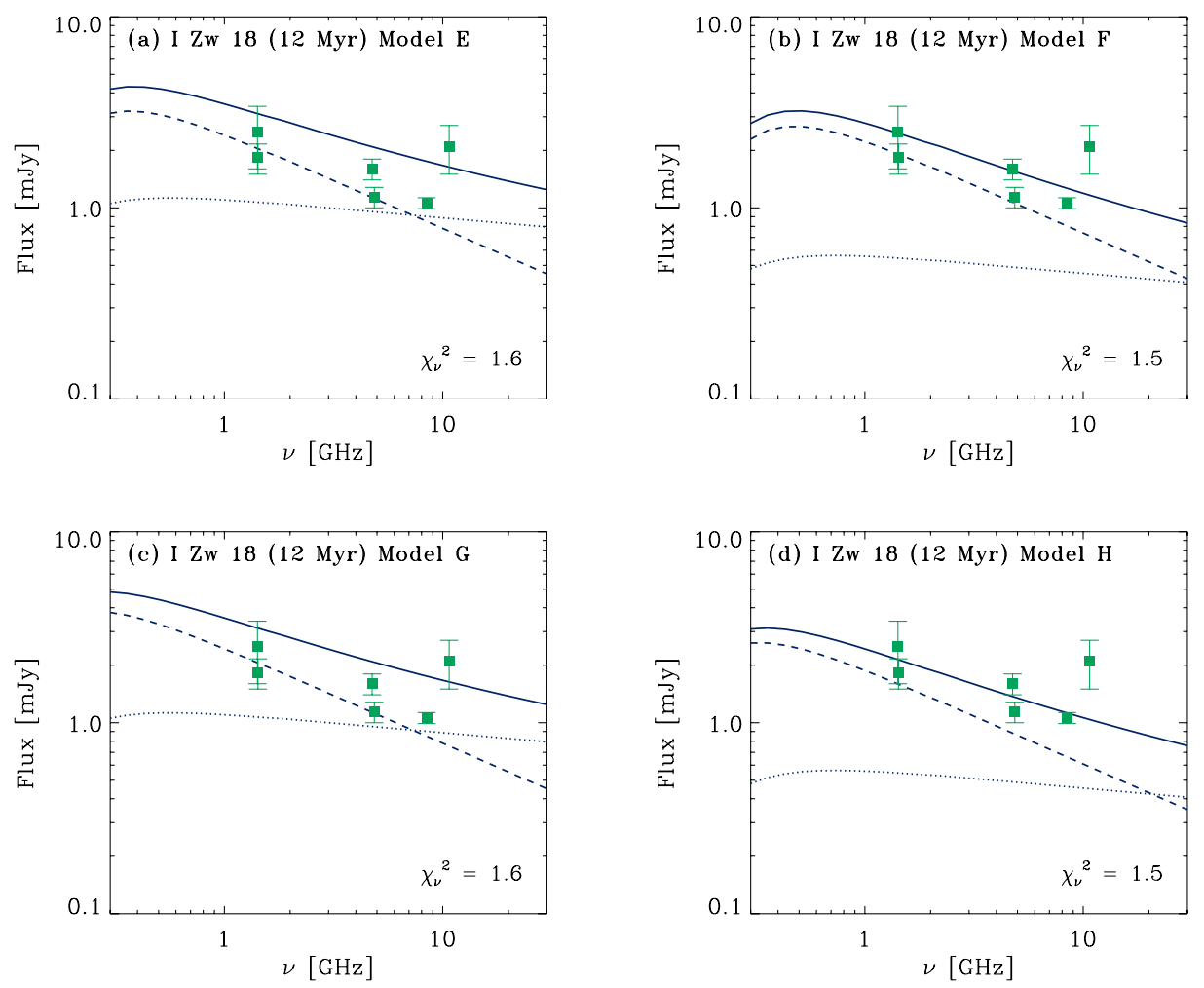

Fig. 4. Radio SED at $t=12 \mathrm{Myr}$ calculated for IZw 18 by using models E, F, G, and H (panels a), b), c), and d), respectively). The dotted and dashed lines represent the thermal and nonthermal components, respectively, and the solid line shows the sum of those two components. The squares are observational data taken from Hunt et al. (2005) and references therein. The reduced $\chi^{2}$ value $\left(\chi_{v}^{2}\right)$ is also shown in each panel.
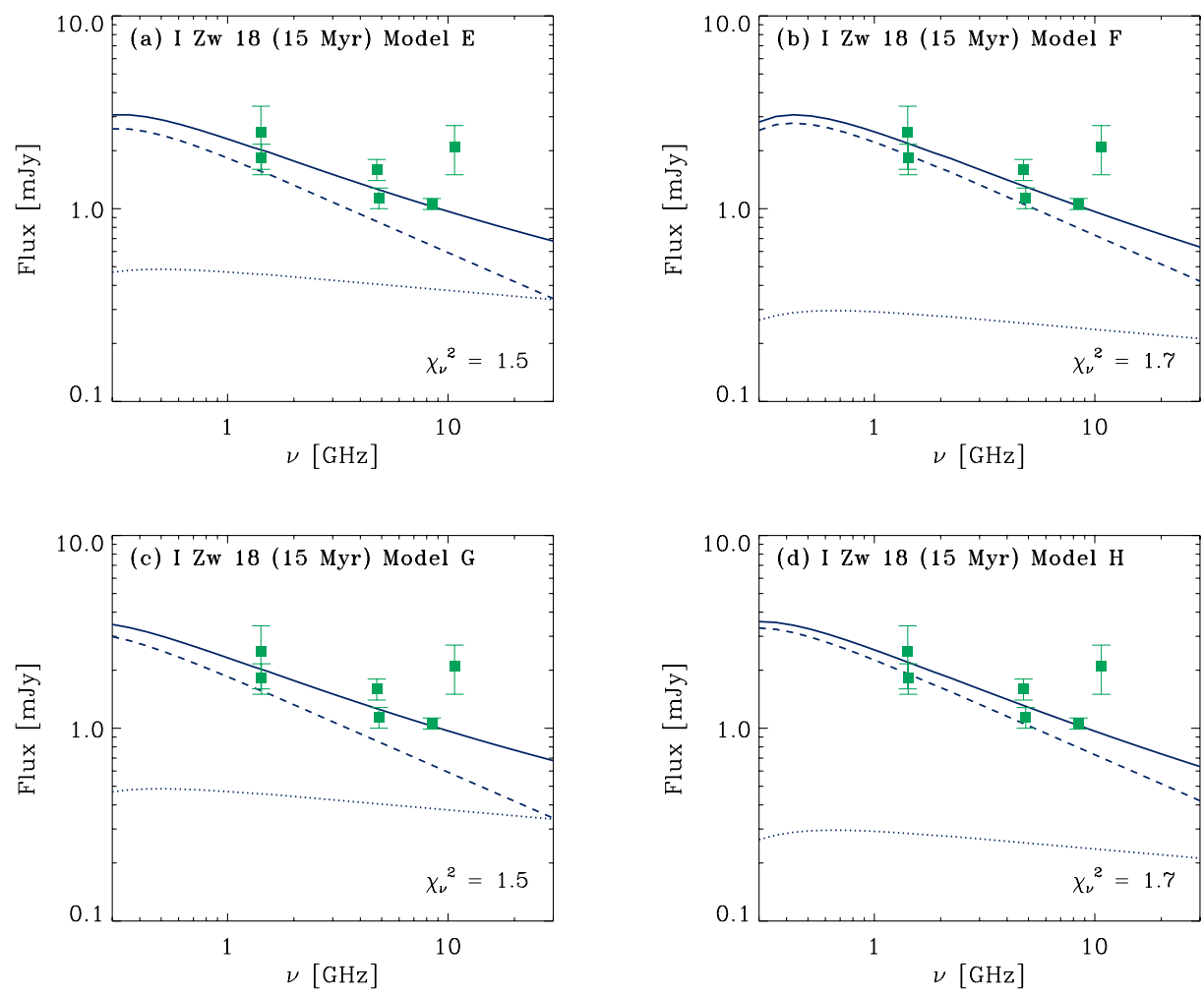

Fig. 5. Same as Fig. 4 but for $t=15$ Myr. 
the fraction of the thermal component is too large to be consistent with observations. Thus, even though there is uncertainty in the age of IZW 18, we can safely conclude that $l_{\mathrm{nt}} \tau_{\mathrm{nt}}$ of IZw 18 is two to five times smaller than that of SBS 0335-052. The typical densities of ionized regions are $\sim 10^{3} \mathrm{~cm}^{-3}$ and $\sim 10^{2} \mathrm{~cm}^{-3}$ in SBS 0335-052 and IZw 18, respectively. We propose in Sect. 4 a scaling relation $l_{\mathrm{nt}} \tau_{\mathrm{nt}} \propto n^{0.35-0.73}$. Since $l_{\mathrm{nt}} \tau_{\mathrm{nt}}=$ $5.8-22 \times 10^{22} \mathrm{~W} \mathrm{~Hz}^{-1} \mathrm{yr}$ has been obtained for SBS 0335-052, the above density scaling relation predicts that $l_{\mathrm{nt}} \tau_{\mathrm{nt}} \sim 1.1-9.9 \times$ $10^{22} \mathrm{~W} \mathrm{~Hz}^{-1}$ yr. The above values derived for I Zw 18 are in this range.

For IZw 18, we obtain the following value for the nonthermal radiative energy over the entire lifetime of a SNR:

$E_{\mathrm{nt}}^{\mathrm{s}}(v)=3.4-9.4 \times 10^{29}\left(\frac{v}{5 \mathrm{GHz}}\right) \mathrm{J} \mathrm{Hz}^{-1}$.

As for SBS 0335-052, this is larger than the "standard" estimate in Eq. (28). The total radio energy per SNR in I Zw 18 becomes

$\mathcal{E}^{\mathrm{p}}(0.1-100 \mathrm{GHz})=1.5-6.0 \times 10^{40} \mathrm{~J}$.

\section{Synchrotron emission and magnetic fields}

Through comparison with observations, we have obtained the radio energy radiated by the nonthermal synchrotron component. In this section, we relate the observationally-derived values to the physical quantities governing the synchrotron radiation.

\subsection{General estimate}

The energy spectrum of energetic electrons radiating synchrotron radiation is assumed to follow a power law as

$N(E) \mathrm{d} E=C E^{-p} \mathrm{~d} E$,

where $N(E) \mathrm{d} E$ is the number density of the energetic electrons with energies between $E$ and $E+\mathrm{d} E$, and $C$ is the normalizing constant. Under this energy spectrum, the energy of the energetic electrons per unit volume, $\varepsilon_{\mathrm{e}}$, is calculated as

$\varepsilon_{\mathrm{e}}=\int_{E_{\min }}^{E_{\max }} E N(E) \mathrm{d} E$,

where $E_{\max }$ and $E_{\min }$ are the maximum and minimum energies of the energetic electrons. Combining Eqs. (34) and (35), we can express the normalizing constant as

$C= \begin{cases}\frac{\varepsilon_{\mathrm{e}}(2-p)}{E_{\max }^{2-p}-E_{\min }^{2-p}} & (p \neq 2) \\ \frac{\varepsilon_{\mathrm{e}}}{\ln \left(E_{\max } / E_{\min }\right)} & (p=2) .\end{cases}$

The emissivity of the synchrotron radiation, $P(v)$, is written as (Ginzburg \& Syrovatskii 1965; Rybicki \& Lightman 1979)

$$
\begin{aligned}
P(v)= & 1.4 \times 10^{-29} a(p) C B^{(p+1) / 2} \\
& \times\left(\frac{v}{6.3 \times 10^{18} \mathrm{~Hz}}\right)^{-(p-1) / 2} \mathrm{~W} \mathrm{~Hz}^{-1} \mathrm{sr}^{-1},
\end{aligned}
$$

where $a(p)$ is a coefficient which depends on the exponent $p$ in the energy spectrum defined in Eq. (34), $C$ is the normalizing constant in Eq. (34), and $B$ is the magnetic field strength in units of gauss.

The spectral index observed in individual SNRs is roughly $\alpha \simeq-(p-1) / 2 \simeq-0.5$ (Clark \& Caswell 1976); i.e., $p \simeq 2$. Since the frequency of the power-law synchrotron radiation ranges at least from $\mathrm{MHz}$ to $\mathrm{GHz}$, we can assume that $\ln \left(E_{\max } / E_{\min }\right) \sim 10$. In any case, because of the logarithmic dependence, the results are not sensitive to the assumed $E_{\max } / E_{\min }$.

The total nonthermal radio luminosity of a region can be calculated by integrating $P(v)$ over all the volume $V$ as

$L_{\mathrm{nt}}^{\text {theory }}=\int_{V} 4 \pi P(v) \mathrm{d} V$

where $L_{\mathrm{nt}}^{\text {theory }}$ is the theoretically estimated nonthermal luminosity of the entire region. Assuming homogeneity of the region and $p=2$, and with $a(2) \simeq 0.103$ (Ginzburg \& Syrovatskii 1965) we obtain

$$
\begin{aligned}
L_{\mathrm{nt}}^{\text {theory }}(v)= & 2.0 \times 10^{19}\left(\frac{\ln \left(E_{\max } / E_{\min }\right)}{10}\right)^{-1} \\
& \times\left(\frac{\mathcal{E}_{\mathrm{e}}}{10^{52} \mathrm{erg}}\right)\left(\frac{B}{10 \mu \mathrm{G}}\right)^{1.5} \\
& \times\left(\frac{v}{5 \mathrm{GHz}}\right)^{-0.5} \mathrm{~W} \mathrm{~Hz}^{-1},
\end{aligned}
$$

where $\mathcal{E}_{\mathrm{e}}$ is the total energy in the volume:

$\mathcal{E}_{\mathrm{e}} \equiv \int_{V} \varepsilon_{\mathrm{e}} \mathrm{d} V$

We assume that a fraction $f_{\mathrm{e}}$ of the input $\mathrm{SN}$ energy is converted to the energy of accelerated energetic electrons:

$\mathcal{E}_{\mathrm{e}} \simeq f_{\mathrm{e}} E_{\mathrm{SN}} \gamma \tau_{\mathrm{e}}$

where $\tau_{\mathrm{e}}$ is the lifetime of energetic electrons. Equating $L_{\mathrm{nt}, \mathrm{p}}^{0}$ (Eq. (10)) and $L_{\mathrm{nt}}^{\text {theory }}$ (Eq. (39)), we obtain the theoretical expression for $l_{\mathrm{nt}} \tau_{\mathrm{nt}}$ at $v=5 \mathrm{GHz}$ as

$$
\begin{aligned}
l_{\mathrm{nt}} \tau_{\mathrm{nt}}= & 2.0 \times 10^{19}\left(\frac{\ln \left(E_{\mathrm{max}} / E_{\mathrm{min}}\right)}{10}\right)^{-1}\left(\frac{f_{\mathrm{e}} E_{\mathrm{SN}}}{10^{47} \mathrm{erg}}\right) \\
& \times\left(\frac{\tau_{\mathrm{e}}}{10^{5} \mathrm{yr}}\right)\left(\frac{B}{10 \mu \mathrm{G}}\right)^{1.5} \mathrm{~W} \mathrm{~Hz}^{-1} \mathrm{yr} .
\end{aligned}
$$

It would be reasonable to assume that $\tau_{\mathrm{nt}}=\tau_{\mathrm{e}}$. However, we retain both quantities since the observational constraint has been applied to $l_{\mathrm{nt}} \tau_{\mathrm{nt}}$.

\subsection{Comparison with SBS 0335-052}

In Sect. 3.1 for SBS 0335-052 we derived the nonthermal radio energy emitted at $5 \mathrm{GHz}$ per $\mathrm{SN}$ as $l_{\mathrm{nt}} \tau_{\mathrm{nt}} \simeq(6-22) \times$ $10^{22} \mathrm{~W} \mathrm{~Hz}{ }^{-1}$ yr. Comparing this value with the theoretical expression given in Eq. (42), we obtain $\left(f_{\mathrm{e}} / 10^{-4}\right)\left(\tau_{\mathrm{e}} / 10^{5} \mathrm{yr}\right)(B / 10 \mu \mathrm{G})^{1.5} \sim(3-11) \times 10^{3}$, where we have assumed $E_{\mathrm{SN}}=10^{51} \mathrm{erg}$ and $\ln \left(E_{\max } / E_{\min }\right)=10$.

Either $B \gg 10 \mu \mathrm{G}$ or $f_{\mathrm{e}} \gg 10^{-4}$ or both. We first investigate the possibility that the magnetic fields could be amplified by turbulence generated from $\mathrm{SNe}$ as proposed by Balsara et al. (2004). We define the ratio of thermal energy to magnetic energy, $\beta \equiv\left(3 n k_{\mathrm{B}} T / 2\right) /\left(B^{2} / 8 \pi\right)$, where $n$ is the particle number density, $k_{\mathrm{B}}$ is the Boltzmann constant, and $T$ is the gas temperature. In SBS 0335-052, we can assume $n \sim 10^{3} \mathrm{~cm}^{-3}$ and $T \sim 2 \times 10^{4} \mathrm{~K}$ (Sect. 3.1). Then, the magnetic field strength becomes $320 \beta^{-1 / 2} \mu \mathrm{G}$. The magnetic field could be amplified in the star-forming region until the magnetic pressure becomes comparable to the thermal pressure (otherwise, the magnetic fields 
would not be confined within the region). Thus, $\beta>1$ would be a reasonable condition. If we conservatively assume that $\beta \sim 10$, we obtain the magnetic field strength in SBS 0335-052 as $100 \mu \mathrm{G}$. The production efficiency of energetic electrons is not well known, but it could be assumed to be of an order of $1 \%$ or more (e.g., Zirakashvili \& Völk 2006). Here we conservatively assume $f_{\mathrm{e}} \sim 0.01$. With the quantities estimated in this paragraph, we predict $\tau_{\mathrm{e}} \sim(6-35) \times 10^{4} \mathrm{yr}$.

This timescale can be interpreted in two ways. One is the radiative lifetime of a SNR. The adiabatic lifetime of a SNR is estimated as $\tau_{\text {ad }} \sim 10^{3} \mathrm{yr}$ if we assume $n_{\mathrm{H}} \sim 10^{3} \mathrm{~cm}^{-3}$ (Eq. (9)). This is much shorter than the above $\tau_{\mathrm{e}}$. However, the SNR continues to expand after the cooling due to the momentum conservation. If we adopt the expansion law of Shull (1980), it finally reaches the sound speed of the ionized medium and the shock disappears after $\sim 10^{5} \mathrm{yr}$. Therefore, the production of energetic electrons in shocked gas may continue on a timescale of $\sim 10^{5} \mathrm{yr}$, which is roughly consistent with $\tau_{\mathrm{e}}$ estimated above. From the expansion law, we can derive $\tau_{\mathrm{e}} \propto n^{-2 / 5}$.

The other interpretation of $\tau_{\mathrm{e}}$ is that it reflects the lifetime of an energetic electron. The energy loss of energetic electrons should be considered at frequencies larger than $v_{\mathrm{m}}$ estimated as

$v_{\mathrm{m}}=\frac{3.1 \times 10^{23}}{B_{\perp}^{3} t^{2}} \mathrm{~Hz}$,

where $B_{\perp}$ is the component of the magnetic field perpendicular to the velocity of the electron, and the time $t$ is measured from the production of the energetic electrons (Ginzburg \& Syrovatskii 1965). We define $\tau_{\mathrm{s}}$ as the timescale on which the energy loss of electrons contributing to the radiation at a frequency $v$ becomes significant. Then, $\tau_{\mathrm{s}}$ can be estimated by inserting $v_{\mathrm{m}}=v$ and $t=\tau_{\mathrm{s}}$ in Eq. (43) as

$\tau_{\mathrm{s}} \simeq 8.0 \times 10^{6}\left(\frac{B_{\perp}}{10 \mu \mathrm{G}}\right)^{-3 / 2}\left(\frac{v}{5 \mathrm{GHz}}\right)^{-1 / 2} \mathrm{yr}$.

If we adopt $B_{\perp} \sim 100 \mu \mathrm{G}$ for SBS 0335-052, we obtain $\tau_{\mathrm{s}} \gtrsim$ $2 \times 10^{5}$ yr at $v \lesssim 10 \mathrm{GHz}$. This is consistent with the above $\tau_{\mathrm{nt}}$. In the following, we adopt an approximation of $B_{\perp} \sim B$.

\subsection{Scaling of $l_{\mathrm{nt}} \tau_{\mathrm{nt}}$}

We investigate both cases for $\tau_{\mathrm{nt}}$. The first is based on the radiative lifetime of a SNR, i.e., $\tau_{\mathrm{nt}}=\tau_{\mathrm{e}} \simeq 10^{5}\left(\mathrm{n} / 10^{3} \mathrm{~cm}^{-3}\right)^{-2 / 5} \mathrm{yr}$, and the second on the lifetime of energetic electrons, i.e., $\tau_{\mathrm{nt}}=\tau_{\mathrm{s}}$ given in Eq. (44). By using Eq. (42), these scaling relations result in the following expression for $l_{\mathrm{nt}} \tau_{\mathrm{nt}}$ :

$l_{\mathrm{nt}} \tau_{\mathrm{nt}} \simeq\left\{\begin{array}{l}2.0 \times 10^{21}\left(\frac{f_{\mathrm{e}} E_{\mathrm{SN}}}{10^{49} \mathrm{erg}}\right)\left(\frac{n}{10^{3} \mathrm{~cm}^{-3}}\right)^{-2 / 5} \\ \times\left(\frac{B}{10 \mu \mathrm{G}}\right)^{1.5} \mathrm{~W} \mathrm{~Hz}^{-1} \mathrm{yr} \\ \text { if } \tau_{\mathrm{SN}}=\tau_{\mathrm{e}}, \\ 3.6 \times 10^{23}\left(\frac{f_{\mathrm{e}} E_{\mathrm{SN}}}{10^{49} \mathrm{erg}}\right) \mathrm{W} \mathrm{Hz}^{-1} \mathrm{yr} \\ \text { if } \tau_{\mathrm{SN}}=\tau_{\mathrm{s}},\end{array}\right.$

where we have assumed that $\ln \left(E_{\max } / E_{\min }\right) \simeq 10$.

It is generally difficult to observe the magnetic field strength in BCDs. If we use $\beta$ (Sect. 4.2), we can express the magnetic field strength in terms of the gas density as $B \simeq 320 \mu \mathrm{G}\left(n / 10^{3} \mathrm{~cm}^{-3}\right)^{1 / 2} \beta^{-1 / 2}$, where we have used $T=2 \times 10^{4} \mathrm{~K}$. A similar observational scaling $\left(B \propto n^{0.48}\right)$ was observationally found by Niklas \& Beck (1997). Inserting this expression into the first case in Eq. (45), we obtain a scaling relation as $l_{\mathrm{nt}} \tau_{\mathrm{nt}}=8.1 \times$ $10^{23}\left(f_{\mathrm{e}} E_{\mathrm{SN}} / 10^{49} \mathrm{erg}\right)\left(n / 10^{3} \mathrm{~cm}^{-3}\right)^{0.35} \beta^{-0.75} \mathrm{~W} \mathrm{~Hz}^{-1}$ yr. If we assume that $f_{\mathrm{e}} E_{\mathrm{SN}}$ does not change among galaxies and that $l_{\mathrm{nt}} \tau_{\mathrm{nt}}$ scales only with density, we obtain $l_{\mathrm{nt}} \tau_{\mathrm{nt}} \simeq$ $\left(l_{\mathrm{nt}} \tau_{\mathrm{nt}}\right)_{\mathrm{SBS}}\left(n / 10^{3} \mathrm{~cm}^{-3}\right)^{0.35}$, where $\left(l_{\mathrm{nt}} \tau_{\mathrm{nt}}\right)_{\mathrm{SBS}}$ is the value obtained for SBS 0335-052 as reported in Table 2.

In the second case in Eq. (45), there is no dependence on magnetic fields and gas density, if $f_{\mathrm{e}} E_{\mathrm{SN}}$ does not depend on those quantities. Thus, we also consider the case where $l_{\mathrm{nt}} \tau_{\mathrm{nt}}$ is independent of $n$.

Finally we propose yet another scaling of the magnetic field strength, assuming that the magnetic field is amplified by SNe, as in the turbulence-driven amplification mechanism proposed by Balsara et al. (2004). Since the SN rate is proportional to the SFR, the energy density of the magnetic fields could scale with the SFR density $\left(\sim \mathrm{SFR} / r^{3}\right)$. Equation (15) implies that the SFR density scales as $n^{3 / 2}$ (this scaling also expresses a Schmidt law; Schmidt 1959). Thus, we obtain $B \propto n^{3 / 4}$. With this scaling relation, and normalizing it to SBS 0335-052, we obtain $l_{\mathrm{nt}} \tau_{\mathrm{nt}} \simeq$ $\left(l_{\mathrm{nt}} \tau_{\mathrm{nt}}\right)_{\mathrm{SBS}}\left(n / 10^{3} \mathrm{~cm}^{-3}\right)^{0.73}$ for the first case in Eq. (45). Since the energy density of energetic electrons is proportional to the $\mathrm{SN}$ rate, this scaling indicates that the magnetic energy density scales with the energy density of energetic electrons.

In summary, the possible scaling of $l_{\mathrm{nt}} \tau_{\mathrm{nt}}$ is summarized by the following three cases:

$l_{\mathrm{nt}} \tau_{\mathrm{nt}}= \begin{cases}\left(l_{\mathrm{nt}} \tau_{\mathrm{nt}}\right)_{\mathrm{SBS}} & \text { Scaling } a, \\ \left(l_{\mathrm{nt}} \tau_{\mathrm{nt}}\right)_{\mathrm{SBS}}\left(n / 10^{3} \mathrm{~cm}^{-3}\right)^{0.35} & \text { Scaling } b, \\ \left(l_{\mathrm{nt}} \tau_{\mathrm{nt}}\right)_{\mathrm{SBS}}\left(n / 10^{3} \mathrm{~cm}^{-3}\right)^{0.73} & \text { Scaling } c\end{cases}$

Indeed, it has recently been found that the $\Sigma-D$ (radio surface brightness to diameter) relation depends on the ambient density of interstellar medium (Arbutina et al. 2004; Arbutina \& Urošević 2005): SNRs with higher ambient density tend to emit more radio energy. Thus, the positive correlation between density and $l_{\mathrm{nt}} \tau_{\mathrm{nt}}$ is supported. We should note that thermal emission may contribute to the radio emission from $\mathrm{SNe}$ especially in dense environments (Urošević \& Pannuti 2005). Thus, our derived values for $l_{\mathrm{nt}} \tau_{\mathrm{nt}}$ may include some contribution from thermal emission. The decomposition of thermal and nonthermal contributions is left for future work.

\subsection{Comparison with $I Z w 18$}

The typical number density of IZw 18 is $n \simeq 100 \mathrm{~cm}^{-3}$ (Sect. 3.2). Comparing Table 2 (model for SBS 0335-052) with Tables 3 and 4 (model for IZw 18 at 12 and $15 \mathrm{Myr}$, respectively), we find that the best model estimate for $\left(l_{\mathrm{nt}} \tau_{\mathrm{nt}}\right)_{\mathrm{IZw}} /\left(l_{\mathrm{nt}} \tau_{\mathrm{nt}}\right)_{\mathrm{SBS}} \simeq 0.13-0.33$, where the subscript "IZw" indicates the value for I Zw 18 . Scaling $a$ would imply no density dependence, so that we would expect $\left(l_{\mathrm{nt}} \tau_{\mathrm{nt}}\right)_{\mathrm{IZw}} \simeq\left(l_{\mathrm{nt}} \tau_{\mathrm{nt}}\right)_{\mathrm{SBS}}$; this is highly inconsistent with our models. If, instead, we use Scaling $b\left(\propto n^{0.35}\right)$, we would derive $\left(l_{\mathrm{nt}} \tau_{\mathrm{nt}}\right)_{\mathrm{IZw}} /\left(l_{\mathrm{nt}} \tau_{\mathrm{nt}}\right)_{\mathrm{SBS}} \simeq$ 0.45 , still a bit larger than our model predictions. Scaling $c$ $\left(\propto n^{0.73}\right)$ would give $\left(l_{\mathrm{nt}} \tau_{\mathrm{nt}}\right)_{\mathrm{IZw}} /\left(l_{\mathrm{nt}} \tau_{\mathrm{nt}}\right)_{\mathrm{SBS}} \simeq 0.19$, which is the most consistent with our results, although Scaling $b$ cannot be rejected with certainty.

Interestingly, the nonthermal luminosity observed in discrete SNRs in ten external galaxies appears to be positively correlated with density (Hunt \& Reynolds 2006), in a way that is consistent with our results. If the time-luminosity integral varies as $n^{0.7}$, 

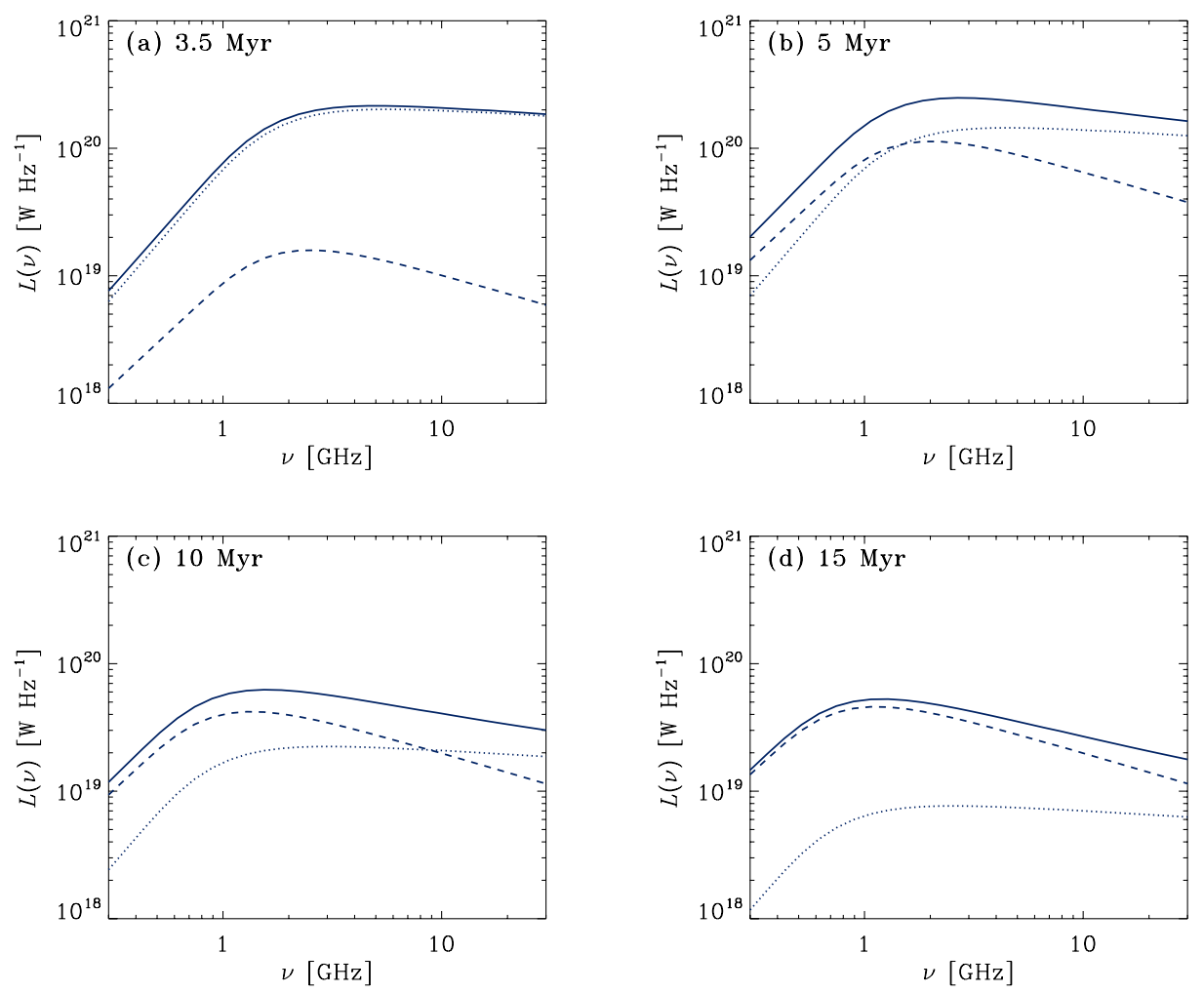

Fig. 6. Time evolution of an "active" radio SED. The same parameters as used to model SBS $0335-052$ are adopted as a representative case for the "active" mode. Panels a), b), c), and d) represent the SEDs at 3.5, 5, 10, and $15 \mathrm{Myr}$, respectively. The dotted and dashed lines represent the thermal and nonthermal components, respectively, and the solid line shows the sum of those two components.

as in the seemingly most likely dependence, then the observed correlation of $l_{n t} \propto n^{1.3}$ implies that the radiative lifetime of a remnant should vary as $n^{-0.6}$. The slope of the $l_{n t}-n$ relation is rather uncertain (Hunt \& Reynolds 2006), but cannot be less than unity. With $l_{n t} \propto n$, the remnant lifetime would vary as $n^{-0.3}$. These values encompass the expansion lifetime dependence $\tau_{\mathrm{e}} \propto$ $n^{-2 / 5}$ and the adiabatic one $\tau_{\mathrm{e}} \propto n^{-9 / 17}$.

\section{The time evolution of the radio continuum}

Cannon \& Skillman (2004) propose the radio spectral index as an age indicator. The radio spectral index is defined by fitting the radio spectrum with a functional form of $v^{\alpha_{\mathrm{f}}}$. In their paper, the observational spectral index is determined with the data at $v=1.4,4.9$, and $8.5 \mathrm{GHz}$. They argue that $\alpha_{\mathrm{f}}>0$ with ages $\$ 1$ Myr because of the free-free absorption. Then the opticallythin thermal spectral index $\left(\alpha_{\mathrm{f}}=-0.1\right)$ appears within a typical lifetime of $\mathrm{H}$ II regions ( $\sim 10 \mathrm{Myr})$ and finally the spectrum steepens because of the increasing contribution from a synchrotron component.

However, we have shown in Sects. 3.1 and 3.2 that the gas density is also important for the shape of the radio spectrum. The dense and compact star-forming region in SBS 0335-052 shows a strong burst of star-formation with strong free-free absorption. We have dubbed this characteristic "active". On the other hand, the diffuse star-forming region in IZw 18 has more mild star-formation with much less absorption. We have called this mode "passive". Since the radio free-free absorption affects the radio spectral index, we can distinguish these two classes of star formation, "active" and "passive", through the radio spectral index. This also means that the age is not the only factor that determines the spectral index; the gas density is also important in its determination.

Here we examine the time evolution of radio spectra, adopting the same parameters as used for SBS 0335-052 and I Zw 18, considering them as representative cases for "active" and "passive", respectively. Model G is adopted here, but the qualitative behavior of the radio spectral index is not much affected by the specific details of the adopted models. We use $l_{\mathrm{nt}} \tau_{\mathrm{nt}}$ estimated for model $\mathrm{G}$ in Tables 2 and 3 for the active and passive cases, respectively. In Figs. 6 and 7, we present the radio SEDs calculated by the models for SBS 0335-052 and IZw 18, respectively. The snapshots at $t=3.5 \mathrm{Myr}$ (soon after the first explosion of SNe at $\simeq 3 \mathrm{Myr}$ ), $5 \mathrm{Myr}, 10 \mathrm{Myr}$, and $15 \mathrm{Myr}$ are shown. We observe that the free-free absorption around $v=1 \mathrm{GHz}$ is prominent in the active case even at $t \gtrsim 5 \mathrm{Myr}$. On the contrary, there is little absorption even at $t \sim 3.5 \mathrm{Myr}$ in the passive case. This indicates that the absorption feature is not a simple indicator of age as suggested by Cannon \& Skillman (2004). The density of the star-forming region is also important for the spectral index around $v=1 \mathrm{GHz}$.

By comparing Figs. 6 and 7, we also see that the nonthermal component becomes comparable to the thermal luminosity earlier in the active case than in the passive case. This mainly comes from the difference in time-integrated radio luminosity of a SN $\left(l_{\mathrm{nt}} \tau_{\mathrm{nt}}\right)$. Thus, we suggest that a nonthermal-dominated radio SED is observed more often in active BCDs than in passive BCDs. This is true if $l_{\mathrm{nt}} \tau_{\mathrm{nt}}$ correlates with gas density. Observations compiled by Hunt \& Reynolds (2006) support this view.

Finally we show the time evolution of radio spectral in$\operatorname{dex} \alpha_{\mathrm{f}}$. The spectral index defined at $v=v_{1}$ and $v_{2}$ is calculated 

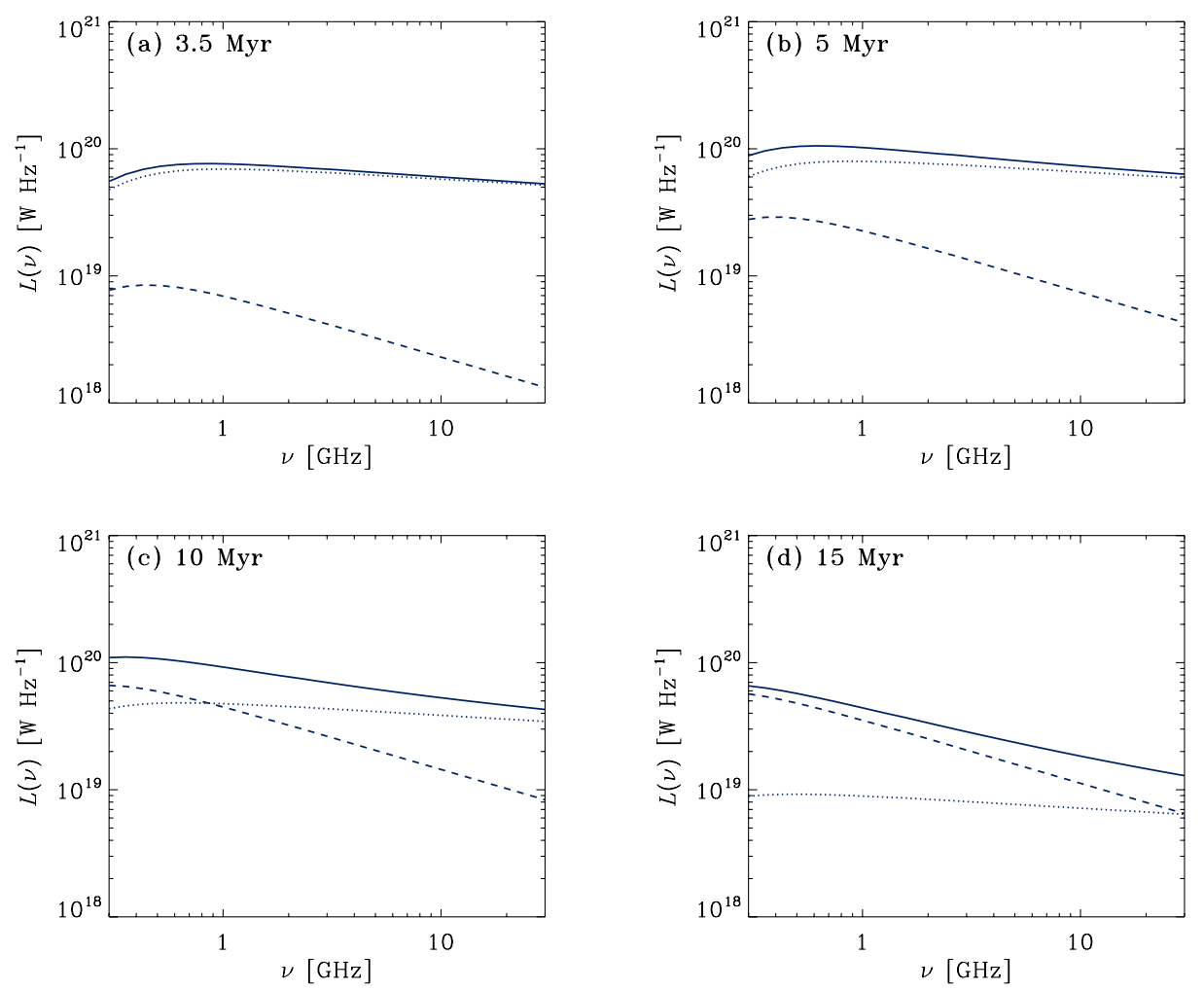

Fig. 7. Same as Fig. 6 but for the "passive" mode, with the IZw 18 model as a representative case.

by the following equation:

$\alpha_{\mathrm{f}}\left(v_{1}, v_{2}\right)=\frac{\log \left[L\left(v_{1}\right) / L\left(v_{2}\right)\right]}{\log \left(v_{1} / v_{2}\right)}$

We put $v_{1}=1.5 \mathrm{GHz}$ and $v_{2}=5 \mathrm{GHz}$ to be consistent with the frequency range often used to determined the spectral index. Figure 8 shows the results. The solid and dotted lines represent the results of the "active" and "passive" models whose spectral shapes are shown in Figs. 6 and 7, respectively. The spectral index depends on age, and it approaches the nonthermal value $(-0.4)$ as the age increases. This confirms the trend suggested by Cannon \& Skillman (2004). More importantly though, the spectral index is not a simple function of age but rather depends strongly on the density of the star-forming region (i.e., "active" and "passive" modes). Indeed the spectral index is positive even at $t=\mathrm{a}$ few Myr in the active mode, while it is always negative in the passive mode.

Another important consideration is that the radio spectrum can be flat (i.e., $\alpha_{\mathrm{f}} \sim 0$ ) even when the contribution from the nonthermal component is significant. Indeed, at $t=5 \mathrm{Myr}$, the nonthermal and thermal components are comparable in the active mode (Fig. 6), and the spectral slope at high frequency indicates the presence of the nonthermal contribution. However, if we define the spectral index by using $v=1.5 \mathrm{GHz}$ and $5 \mathrm{GHz}$, the spectral index becomes $\sim 0$. This spectral index could be misinterpreted as thermal. Thus, in order to avoid such a misinterpretation, it is important to derive a spectral index at $v \gtrsim 5 \mathrm{GHz}$. Our results are also applicable to high- $z$ samples (e.g., Oyabu et al. 2005) in the future as shown below.

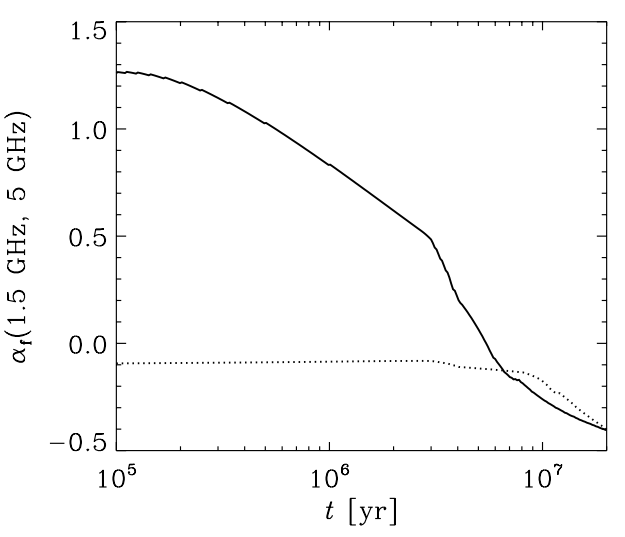

Fig. 8. Time evolution of the radio spectral index defined at $1.5 \mathrm{GHz}$ and $5 \mathrm{GHz}[\alpha(1.5 \mathrm{GHz}, 5 \mathrm{GHz})]$. The solid and dotted lines show the results of the "active" and "passive" models (same as Figs. 6 and 7, respectively).

\subsection{Implications for primeval galaxies}

High- $z$ primeval galaxies tend to have a high density; they are formed and evolve in deep gravitational potentials (e.g., Padmanabhan 1993). Therefore, the gas in such primeval objects may have high pressure, and the star-forming regions may be expected to mimic the active mode (Hirashita \& Hunt 2004). Indeed, the number density of gas in high- $z$ galaxies is estimated as $\gtrsim 10^{3} \mathrm{~cm}^{-3}$ (Norman \& Spaans 1997), and is similar to that of SBS 0335-052, typical of the active class. Therefore, the nonthermal component could dominate on short timescales after the onset of star formation while the radio emission is optically thick 
at $v \lesssim 1 \mathrm{GHz}$. The dense environment could also aid amplification of magnetic fields as proposed by the scaling relations in Sect. 4.3.

This paper also has implications for the production of highenergy electrons. It would be reasonable to consider that the production occurs on an expansion timescale SNRs, since the shocks associated with SNRs can be responsible for the acceleration of electrons (e.g., Axford 1981). The lifetime of nonthermal radiation per $\mathrm{SN}$ is determined by the minimum of two timescales: the lifetime of the shock and the synchrotron loss timescale.

\section{Conclusions}

In order to investigate the time evolution of the radio SEDs of metal-poor star-forming galaxies, we have constructed a radio SED model by treating the thermal and nonthermal components consistently with the star formation history. In particular, the duration and luminosity of the nonthermal radio emission from supernova remnants (SNRs) has been constrained by using the observational radio SEDs of SBS 0335-052 and I Zw 18, both of which are reasonable proxies for young galaxies in the nearby universe. In SBS 0335-052, the typical radio energy emitted per SNR over its lifetime is estimated to be $\mathcal{E}(0.1-100 \mathrm{GHz}) \sim 8-32 \times 10^{47} \mathrm{erg}$. In I Zw 18 , another representative of metal-poor star-forming galaxies, we find that $\mathcal{E}(0.1-100 \mathrm{GHz}) \sim 2-6 \times 10^{47} \mathrm{erg}$. Both estimates are significantly larger than previous estimates based on the $\Sigma-D$ relation. These values of the two "template" galaxies can be simultaneously explained by a simple density scaling relation of $B \propto n^{0.35-0.73}$, indicating that the magnetic pressure scales with the gas pressure, or that the magnetic fields are amplified by $\mathrm{SNe}$, or both.

We have also predicted the time dependence of the radio spectral index and of the radio SED itself, for both the active (SBS 0335-052) and passive (IZw 18) cases. These models enable us to roughly age date and classify radio spectra of star-forming galaxies into active/passive classes. Since the radio emission around $v \lesssim 1 \mathrm{GHz}$ can be affected by the free-free absorption especially in the active class, the spectral index defined at $v \gtrsim 5 \mathrm{GHz}$ should be used to estimate the contribution from the nonthermal component. On the other hand, the free-free absorption feature around $v \sim 1 \mathrm{GHz}$ could be used to select the active class.

Acknowledgements. We thank the anonymous referee for useful comments which improved this paper considerably. We are grateful to T. Yoshida, D. Urošević, Y. Kato, T. Kamae, and H. Kamaya for stimulating discussions on supernovae, high energy phenomena, and magnetic fields. H.H. has been supported by the University of Tsukuba Research Initiative and by Grants-in-Aid for Scientific Research of the Ministry of Education, Culture, Sports, Science and Technology (Nos. 18026002 and 18740097). This research has made use of the NASA/IPAC Extragalactic Database (NED), which is operated by the Jet Propulsion Laboratory, California Institute of Technology, under contract with the National Aeronautics and Space Administration (NASA). We have relied upon NASA's Astrophysics Data System Abstract Service (ADS).

\section{Appendix A: Analysis of expanding $\mathrm{H}$ ॥ regions}

We derive some approximate analytic solutions for expanding ionized regions based on Eqs. (23) and (24). By combining these two equations, we obtain

$$
\frac{\mathrm{d} r_{\mathrm{i}}}{\mathrm{d} t}=C_{\mathrm{II}}\left(\frac{r_{\mathrm{i} 0}}{r_{\mathrm{i}}}\right)^{3 / 4} \text {. }
$$

Since we estimate $r_{\mathrm{i} 0}$ by the Strömgren radius under the current stellar luminosity and the gas density of the neutral medium outside the ionized region, we obtain

$r_{\mathrm{i} 0}=\mathcal{A} \frac{\dot{N}_{\mathrm{ion}}(t)^{1 / 3}}{n_{\mathrm{H} 0}^{2 / 3}}$,

where

$\mathcal{A} \equiv\left(\frac{3}{4 \pi(1.08) \alpha^{(2)}}\right)^{1 / 3}$.

The factor 1.08 comes from the correction for ionized helium (Eq. (5)). By combining Eqs. (A.1)-(A.3), we obtain

$\frac{\mathrm{d} r_{\mathrm{i}}}{\mathrm{d} t}=C_{\mathrm{II}} \mathcal{A}^{3 / 4} n_{\mathrm{H} 0}^{-1 / 2}\left[\dot{N}_{\mathrm{ion}}(t)\right]^{1 / 4} r_{\mathrm{i}}^{-3 / 4}$

Solving this equation, we obtain

$\frac{4}{7} r_{\mathrm{i}}^{7 / 4}=C_{\mathrm{II}} \mathcal{A}^{3 / 4} n_{\mathrm{H} 0}^{-1 / 2} \int_{0}^{t} N_{\mathrm{ion}}\left(t^{\prime}\right)^{1 / 4} \mathrm{~d} t^{\prime}$,

where we have assumed that $r_{\mathrm{i}}(t=0)=0$. If we assume that the SFR is constant and that the death of stars is negligible, $\dot{N}_{\text {ion }}$ is almost proportional to $t$, and we can predict that $r_{\mathrm{i}} \propto t^{5 / 7}$. This relation, combined with Eqs. $(23)\left(\rho_{\mathrm{II}} \propto\left(\mathrm{d} r_{\mathrm{i}} / \mathrm{d} t\right)^{2}\right)$, indicates that $\rho_{\mathrm{II}} \propto t^{-4 / 7}$. The emission measure evolves as $E M \propto \rho_{\mathrm{II}}^{2} r_{\mathrm{i}} \propto t^{-3 / 7}$. In spite of this rough derivation, those relations explain the behaviors depicted in Fig. 1. In the burst SFR (Fig. 1), the emission measure is particularly deviates from the predicted power-law evolution. This is because the first increase of $r_{\mathrm{i}}$ is due to increase of the Strömgren radius itself, not due to dynamical expansion.

\section{References}

Aloisi, A., Tosi, M., \& Greggio, L. 1999, AJ, 118, 302 Arbutina, B., \& Urošević, D. 2005, MNRAS, 360, 76

Arbutina, B., Urošević, D., Stanković, M., \& Tešić, Lj. 2004, MNRAS, 350, 346 Axford, W. I. 1981, in Origin of Cosmic Rays, IAU Symp. 94, ed. G. Setti, G. Spada, \& A. W. Wolfendale (Dordrecht: Reidel), p. 339

Balsara, D. S., Kim, J., Mac Low, M.-M., \& Mathews, G. J. 2004, ApJ, 617, 339 Becker, R. H., White, R. L., \& Helfand, D. J. 1995, ApJ, 450, 559

Biermann, P. 1976, A\&A, 53, 295

Cannon, J. M., \& Skillman, E. D. 2004, ApJ, 610, 772

Cannon, J. M., Skillman, E. D., Garnett, D. R., \& Dufour, R. J. 2002, ApJ, 565, 931

Cannon, J. M., Walter, F., Skillman, E. D., \& van Zee, L. 2005, ApJ, 621, L21

Clark, D. H, \& Caswell, J. L. 1976, MNRAS, 174, 267

Condon, J. J. 1992, ARA\&A, 30, 575

Condon, J. J., Cotton, W. D., Greisen, E. W., et al. 1998, AJ, 115, 1693

Dale, D. A., Helou, G., Neugebauer, G., et al. 2001, AJ, 122, 1736

Dunne, L., Eales, S., Ivison, R., et al. 2003, Nature, 424, 285

Elmegreen, B. G. 2000, ApJ, 530, 277

Ginzburg, V. L., \& Syrovatskii, S. I. 1965, ARA\&A, 3, 297

Has-Hesse, J. M., \& Kunth, D. 1999, A\&A, 349, 765

Helou, G., \& Bicay, M. D. 1993, ApJ, 415, 93

Hirashita, H., \& Hunt, L. K. 2004, A\&A, 421, 555

Hirashita, H., Hunt, L. K., \& Ferrara, A. 2002, MNRAS, 330, L19

Hunt, L. K., Dyer, K. K., \& Thuan, T. X. 2005, A\&A, 436, 837

Hunt, L. K., \& Reynolds, S. P. 2006, to appear in the Proceedings of Cosmic Magnetism, Bologna, 2005, ed. Beck, R., Brunetti, E., \& Feretti, L.

Hunt, L. K., Vanzi, L., \& Thuan, T. X. 2001, A\&A, 377, 66

Hunt, L. K., Hirashita, H., Thuan, T. X., Izotov, Y. I., \& Vanzi, L. 2003a, in Proceedings of Galaxy Evolution: Theory and Observations, ed. V. Avila-Reese, C. Firmani, C. Frenk, \& C. Allen, RevMexAA SC [arXiv: astro-ph/0310865]

Hunt, L. K., Thuan, T. X., \& Izotov, Y. I. 2003b, ApJ, 588, 281

Hunt, L. K., Dyer, K. K., Thuan, T. X., \& Ulvestad, J. S. 2004, ApJ, 606, 853 (H04)

Hopkins, A. M., Schulte-Ladbeck, R. E., \& Drozdovsky, I. O. 2002, AJ, 124, 862 
Inoue, A. K., Hirashita, H., \& Kamaya, H. 2000, AJ, 120, 2415

Ilovaisky, S. A., \& Lequeux, J. 1972, A\&A, 20, 347

Izotov, Y. I., \& Thuan, T. X. 1999, ApJ, 511, 639

Izotov, Y. I., \& Thuan, T. X. 2004, ApJ, 602, 200

Izotov, Y. I., Chaffee, F. H., Foltz, C. B., et al. 1999, ApJ, 527, 757

Izotov, Y. I., Chaffee, F. H., Foltz, C. B., et al. 2001, ApJ, 566, 222

Klein, U., Weiland, H., \& Brinks, E. 1991, A\&A, 246, 323

Kozasa, T., Hasegawa, H., \& Nomoto, K. 1989, ApJ, 344, 325

Lequeux, J., \& Viallefond, F. 1980, A\&A, 91, 269

Lisenfeld, U., \& Ferrara, A. 1998, ApJ, 496, 145

Mannucci, F., Della Valle, M., Panagia, N., et al. 2005, A\&A, 433, 807

McKee, C. F. 1989, in Interstellar Dust, ed. L. J. Allamandola \& A. G. G. M. Tielens (Dordrecht: Kluwer), IAU Symp., 135, 431

Nagata, H., Shibai, H., Takeuchi, T. T., \& Onaka, T. 2002, PASJ, 54, 695

Niklas, S., \& Beck, R. 1997, A\&A, 320, 54

Norman, C. A., \& Spaans, M. 1997, ApJ, 480, 145

Nozawa, T., Kozasa, T., Umeda, H., et al. 2003, ApJ, 598, 785

Östlin, G. 2000, ApJ, 535, L99

Owens, A. J., \& Jokipii, J. R. 1977, ApJ, 215, 685

Oyabu, S., Yun, M. S., Murayama, T., et al. 2005, AJ, 130, 2019

Padmanabhan, T. 1993, Structure formation in the universe (Cambridge: Cambridge University Press)

Plante, S., \& Sauvage, M. 2002, AJ, 124, 1995

Recchi, S., Matteucci, F., D'Erdole, A., \& Tosi, M. 2004, A\&A, 426, 37
Roussel, H., Helou, G., Beck, R., et al. 2003, ApJ, 593, 733

Rybicki, G. B., \& Lightman, A. P. 1979, Radiative Processes in Astrophysics (New York: Wiley)

Salpeter, E. E. 1955, ApJ, 121, 161

Schaerer, D. 2002, A\&A, 382, 28

Schmidt, M. 1959, ApJ, 129, 243

Sedlmayr, E. 1989, in Interstellar Dust, ed. L. J. Allamandola \& A. G. G. M. Tielens (Dordrecht: Kluwer), IAU Symp., 135, 467

Shull, J. M. 1980, ApJ, 237, 769

Skillman, E. D., \& Kennicutt, R. C., Jr. 1993, ApJ, 411, 655

Spitzer, L., Jr. 1978, Physical Processes in the Interstellar Medium (New York: Wiley)

Takeuchi, T. T., Hirashita, H., Ishii, T. T., Hunt, L. K., \& Ferrara, A. 2003, MNRAS, 343, 839

Thuan, T. X., Izotov, Y. I., \& Lipovetsky, V. A. 1997, ApJ, 477, 661

Thuan, T. X., Sauvage, M., \& Madden, S. 1999, ApJ, 516, 783

Tinsley, B. M. 1980, Fundam. Cosmic Phys., 5, 287

Todini, P., \& Ferrara, A. 2001, MNRAS, 325, 726

Ulvestad, J. S. 1982, ApJ, 259, 96

Urošević, D., \& Pannuti, T. G. 2005, Astropart. Phys., 23, 577

van Zee, L., Westpfahl, D., Haynes, M. P., \& Salzer, J. J. 1998, AJ, 115, 1000

Vanzi, L., Hunt, L. K., Thuan, T. X., \& Izotov, Y. I. 2000, A\&A, 363, 493

Woltjer, L. 1972, ARA\&A, 10, 129

Zirakashvili, V. N., \& Völk, H. J. 2006, ApJ, 636, 140 